Canadian

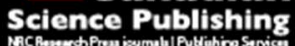

Canadian Journal of Microbiology Revue canadienne de de microbiologie

\title{
Effects of several in-feed antibiotic combinations on the abundance and diversity of fecal microbes in weaned pigs
}

\begin{tabular}{|r|l|}
\hline Journal: & Canadian Journal of Microbiology \\
\hline Manuscript ID & cjm-2016-0681.R1 \\
\hline Danuscript Type: & Article \\
\hline Complete List of Authors: & $\begin{array}{r}\text { Li, Huizhi; Nanjing Agricultural University, College of Animal Science and } \\
\text { Techonolgy } \\
\text { Liang, Tingting; Nanjing Agricultural University, College of Animal Science } \\
\text { and Techonolgy } \\
\text { Chu, Qingpo; Nanjing Agricultural University, College of Animal Science } \\
\text { and Techonolgy } \\
\text { Xu, Feilong; Nanjing Agricultural University, College of Animal Science and } \\
\text { Techonolgy } \\
\text { Li, Yuan; Nanjing Agricultural University, College of Animal Science and } \\
\text { Techonolgy } \\
\text { Fu, Lingling; Nanjing Agricultural University, College of Animal Science and } \\
\text { Techonolgy } \\
\text { Zhou, Bo; Nanjing Agricultural University, College of Animal Science and } \\
\text { Technology }\end{array}$ \\
\hline Keyword: & \begin{tabular}{l} 
16S rRNA gene, antibiotic combination, fecal microbes, Illumina, pig \\
\hline
\end{tabular} \\
\hline
\end{tabular}


Effects of several in-feed antibiotic combinations on the abundance and diversity of fecal microbes in weaned pigs

Authors: Huizhi Li, Tingting Liang, Qingpo Chu, Feilong Xu, Yuan Li, Lingling Fu and Bo Zhou ${ }^{*}$

College of Animal Science and Technology, Nanjing Agricultural University, Nanjing 210095, P. R. China

*Corresponding author: College of Animal Science and Technology, Nanjing Agricultural University, Nanjing 210095, P.R. China.

Tel: +86 25 84395362; Fax: +86 25 84395314; E-mail: zhoubo@njau.edu.cn

All authors disclose any conflict of interest 


\section{Abstract:}

To characterize the effects of several subtherapeutic antibiotic combinations on the abundance and diversity of fecal microbes, 400 weaned pigs were selected and randomly assigned to 8 groups, where they were continuously fed different antibiotic combinations for 28 days. Then, a total of 48 pigs were randomly selected to individually collect the feces for DNA extraction and 16S rRNA high-throughput sequencing. Compared with the pigs without antibiotic administration, the diversities of fecal microbes were decreased in the pigs fed chlortetracycline (CTC), olaquindox (OLA) and enramycin (ER)/ virginiamycin (VIR), where the relative abundance of the phymum Bacteroidetes and the genus Prevotella were increased. Compared with the pigs without antibiotic administration, the relative abundance of the phymum Proteobacteria and the genus Succinivibrio were decreased in the pigs fed CTC, zinc bacitracin (ZB) and colistin sulphate (CT), a mixture of CTC, ZB and CT, CTC and OLA, or a mixture of CTC, OLA and ER. Alpha-diversity and $\beta$-diversity were decreased $(P<0.05)$ in the pigs fed ZB and CT, or a mixture of CTC, OLA and ER/VIR. This study provides experimental data to deepen our understanding on the effects of antibiotic combinations on intestinal microbes.

Keywords: 16S rRNA gene; antibiotic combination; fecal microbes; Illumina; pig 


\section{Introduction}

The use of subtherapeutic antibiotic in-feed has been reported to increase growth rates and feed efficiency of pigs (Cromwell 2002; van der Fels-Klerx et al. 2011). However, in-feed antibiotics have worsen the already alarming scenario of antibiotic resistance (Ahmed et al. 2014). In pig production, antibiotic combinations are typically used in-feed as antibiotic growth promoters (AGPs) (Cromwell 2002) in some countries, although in parts of the world the procedure of using antimicrobials for growth promotion is banned. However, the impact of in-feed antibiotic combinations on the relative abundance and diversity of intestinal microbiota in pigs has not been well studied.

There are several antimicrobial agents permitted for use in pig production in China, such as chlortetracycline (CTC), zinc bacitracin (ZB), colistin sulphate $(\mathbf{C T})$, olaquindox (OLA), enramycin (ER), and virginiamycin (VIR). CTC belongs to the tetracycline class, which are broad-spectrum agents, exhibiting antibacterial activity against bacteria, mycoplasmas, chlamydiae, and protozoan parasites (Chopra and Roberts 2001). ZB, a water-soluble and non-toxic antibiotic, is active chiefly against gram-positive bacteria, such as streptococcal and staphylococcal infections (Johnson et al. 1945). In chickens, ZB in the diet significantly reduced the number of coliform bacteria in the ileum (Engberg et al. 2000) and the abundance of lactobacilli in the caeca (Gong et al. 2008). COL is active chiefly against gram-negative organisms by alteration of permeability of membrane (Nakajima and Kawamata 1965). Until now OLA, a member of quinoxaline-1,4-dioxides derivatives with antibacterial activity 
against bacteria and toxicity, is still used in China for promoting pig growth (Chen et al. 2009). ER has antibacterial activity against gram-positive bacteria (Kawakami et al. 1971). VIR may reduce the concentration of gram-positive bacteria and increase the apparent ileal digestibility of amino acid in pigs (Stewart et al. 2010). As an alternative to antibiotics, Macleaya cordata extract contains considerable amounts of sanguinarine, chelerythrine, dihydrochelerythrine, dihydrosanguinarine, allocryptopine, and protopine, which have broad antimicrobial activity as well as antiinflammatory properties (Kosina et al. 2010).

In the mammalian gastrointestinal tract, a large and diverse microbial population plays a major role in health and nutrient digestion (Kim et al. 2012; Yatsunenko et al. 2012). Previous studies showed that tylosin and CTC altered the gut microbial community of pigs (Kim et al. 2012; Rettedal et al. 2009). Nevertheless, Castillo et al. (2006) reported that the types of feed additives had little impact on bacteria counts in gastrointestinal tracts but changed the community structure. A mixture of CTC, sulfamethazine, and penicillin (APS250) decreased the relative abundance of Bacteroidetes (Looft et al. 2012). However, administration of carbodox increases the relative abundance of genus Roseburia, Prevotella, and Fecalibacterium (Looft et al. 2014b).

We hypothesized that antibiotics can differentially affect the intestinal microflora of pigs because of their different antimicrobial mechanisms. The objective of this study was to investigate effects of in-feed antibiotic combinations on the abundance and diversity of fecal microbes in weaned pigs. In the present study, we sequenced the 
V3-V4 regions of 16S rRNA genes in the Bacteria/Archaea to investigate the impacts of antibiotic combinations on pig fecal microbiota using Illumina HiSeq 2500 sequencer.

\section{Materials and Methods}

\section{Pigs and fecal collection}

The present study was reviewed and approved by animal use committee of Nanjing Agricultural University. The present study was carried out from October to November in 2014 at Yong-kang Agricultural and Animal Husbandry Co., Ltd. in Jiangsu Province, China $\left(31.74^{\circ} \mathrm{N}\right)$.

Four hundred 35-day-old weaned pigs (Duroc $\times$ Landrace $\times$ Large White, body weight $12.1 \pm 0.3 \mathrm{~kg}$ ) were selected and randomly assigned to eight treatments:

(Table 1). There were three replicates (pens) in each treatment and 16-17 pigs per pen. All the experimental pigs were raised in the same room. Each pen $(1.7 \times 3.5 \mathrm{~m})$ was equipped with a feeder (1.2 m long) and two nipple drinkers to allow ad libitum access to feed and water. After 28 days of continuous antibiotics administration, a total of 48 pigs (two pigs per pen, 1 barrow and 1 female) were randomly selected to individually collect the fresh feces into $2 \mathrm{~mL}$ centrifugal tubes and stored at $-20^{\circ} \mathrm{C}$ until DNA extraction.

\section{DNA extraction and high-throughput sequencing}

Microbial genomic DNA extraction was performed using a bead-beating and CTAB (cetyltrimethyl ammonium bromide) method referring to our previous study (Li et al. 2016). The concentration of extracted genomic DNA was measured using a 
spectrophotometer (NanoDrop 2000, Thermo, Waltham, MA, USA).

Based on previous studies (Caporaso et al. 2011; Kim et al. 2012; Youssef et al. 2009), the V3-V4 hypervariable regions of the 16S rRNA gene were amplified and then sequenced, the broadly conserved primer 314F (CCT AYG GGR BGC ASC AG) and 806R (GGA CTA CNN GGG TAT CTA AT) targeting the Bacteria and Archaea 16S V3-V4 regions were selected for the microbial community structure analysis (Caporaso et al. 2011). The PCR amplifications of the 16S rRNA gene were performed in a total volume of $50 \mu \mathrm{L}$ containing $25 \mu \mathrm{L}$ rTaq DNA polymerase (Takara Biotech, Co., Ltd., Dalian, China), $1.0 \mu \mathrm{M}$ of each primer, $120 \mathrm{ng}$ template DNA. The thermal cycling conditions were as follows: $95^{\circ} \mathrm{C}$ for $5 \mathrm{~min}, 35$ cycles of $95{ }^{\circ} \mathrm{C}$ for 30 $\mathrm{s}$, temperatures of annealing for $30 \mathrm{~s}$, and $72^{\circ} \mathrm{C}$ for $45 \mathrm{~s}$, followed by $72{ }^{\circ} \mathrm{C}$ for $7 \mathrm{~min}$. All PCR reactions were carried out using Phusion High-Fidelity PCR Master Mix (New England Biolabs). Mix same volume of $1 \times$ loading buffer (contained SYB green) with PCR products and operate electrophoresis on $2 \%$ agarose gel for detection. Samples with bright main strip between 400-450bp were chosen for further experiments. Only PCR products without primer dimers and contaminant bands were used for the high-throughput sequencing. Barcoded V3-V4 Amplicons were sequenced using the Illumina HiSeq platform (Illimina, USA) at Novogene Institute (Beijing, China) according to the manufacturer's instructions.

Based on their unique barcode, paired-end reads were assigned to samples, truncated by cutting off the barcode and primer sequence, and merged using FLASH software (V1.2.7). According to the QIIME (V1.7.0) quality controlled process, 
quality filtering on the raw tags were performed to obtain the clean tags. The tags were compared with the reference database Gold using UCHIME algorithm to detect and remove chimera sequences. Then the Effective Tags finally obtained. Sequences analysis were completed using Uparse. Sequences with $\geq 97 \%$ similarity were assigned to the same OTUs. For each OUT, representative sequence was screened for further annotation. The taxonomic information of each representative sequence was annotated using the GreenGene Database based on RDP classifier algorithm. In order to study phylogenetic relationship between OTUs and dominant species in different samples or groups, multiple sequence alignments were conducted using the MUSCLE software. Abundance information of OTU was normalized using a standard of sequence number corresponding to the sample with the least sequences. Subsequent analysis of alpha diversity and beta diversity were all performed basing on this output normalized data using QIIME and displayed with R software. Cluster analysis was preceded by principal component analysis (PCA), which was used to reduce the dimension of the original variables using the FactoMineR package and ggplot2 package in R software. Principal Coordinate Analysis (PCoA) was performed to get principal coordinates and visualize from complex, multidimensional data. A distance matrix of weighted or unweighted unifrac among samples obtained before was transformed to a new set of orthogonal axes, by which the maximum variation factor is demonstrated by the first principal coordinate, the second maximum one by the second principal coordinate, and so on. PCoA analysis was displayed by WGCNA package, stat packages and ggplot2 package in R software. Unweighted Pair-group 
Method with Arithmetic Means (UPGMA) Clustering was performed as a type of hierarchical clustering method to interpret the distance matrix using average linkage and was conducted by QIIME software.

\section{Results}

\section{Sequence analysis}

A total of 3,861,058 raw reads were obtained from the Illumina HiSeq sequencing. Following quality control to remove chimera, 2,535,291 high quality sequences with an average length of $414 \mathrm{bp}$ were remained (Table S1). The average number of sequences per sample was 52,819. Fig. S1 shows the rarefaction curves of treatments of antibiotic combinations that indicate a high level of microbial diversity. Based on $97 \%$ species similarity, the superior quality sequences were clustered into operational taxonomic units (OTUs) using the de novo method. The removal of low abundance OTUs $(<0.005 \%)$ yielded a total of 1166 OTUs for analysis. Good's coverage of all samples was $\geq 99.7 \%$ (Table S2).

\section{Microbial abundance of the pig fecal microbiota}

At the phylum level, the 10 most relatively abundant bacterial phyla were Firmicutes, Bacteroidetes, Proteobacteria, Euryarchaeota, Spirochaetes, Tenericutes, Cyanobacteria, Actinobacteria, Elusimicrobia, and Fibrobacteres (Table S3). Firmicutes and Bacteroidetes dominated, accounting for $54.2 \%$ and $40.4 \%$ of sequences, respectively. Only $1.2 \%$ of sequences could not be classified at the phylum level. Compared to the group without antibiotics, the relative abundances of Firmicutes were increased $(P<0.05)$ in the groups fed with CTC, or a mixture of ZB 
and $\mathrm{CT}$, and decreased $(P<0.05)$ in the group fed with a mixture of CTC, OLA and VIR. The relative abundances of Bacteroidetes were increased $(P<0.01)$ in the groups fed with a mixture of CTC, OLA and ER, and a mixture of CTC, OLA and VIR, and the relative abundances of Proteobacteria were decreased in the groups fed with CTC $(P<0.05)$, a mixture of ZB and COL $(P<0.01)$, a mixture of CTC, ZB and $\operatorname{COL}(P<0.05)$, a mixture of CTC and OLA $(P<0.05)$, and a mixture of CTC, OLA and ER $(P<0.01)$ (Fig. 1a and Table 2).

Prevotella was the most relatively abundant genus accounting for $18.89 \%$ of all sequences followed by Roseburia (8.45 \%), [Prevotella] (5.39\%), Faecalibacterium (4.12\%), Succinivibrio (3.17\%), Lachnospira (3.12\%), Ruminococcus (2.97\%), Oscillospira (2.81\%), Coprococcus (0.95\%), CF231 (0.93\%), Phascolarctobacterium (0.90\%), p-75-a5 (0.57\%), Parabacteroides $(0.54 \%)$, Bacteroides (0.52\%), and Methanobrevibacter (0.46\%).

The sequences were assigned into 118 different genera, with an average of $43.3 \%$ of sequences being unclassified at the genus level. Compared to the control group, the relative abundances of Prevotella increased $(P<0.01)$ in the groups fed with a mixture of CTC, OLA and ER, and a mixture of CTC, OLA and VIR. Compare with the control group, the relative abundances of Succinivibrio were decreased in the groups fed with CTC $(P<0.05)$, a mixture of ZB and COL $(P<0.01)$, a mixture of CTC, ZB and COL $(P<0.05)$, a mixture of CTC and OLA $(P<0.05)$, and a mixture of CTC, OLA and ER $(P<0.05)$. The relative abundance of Faecalibacterium was less $(P<0.01)$ in the group fed with a mixture of CTC, OLA and ER than in the 
control group (Fig. 1b and Table 3).

The distribution of predominant bacterial OTUs in which the relative abundance was over 1\% is shown in Fig. S2 and the OTU Taxonomy is shown in Table S4.

Among the 1,168 OTUs identified in the present study, 62 OTUs were significantly ( $P$ $<0.01$ ) affected by antibiotic combinations (Fig. S3). Among them, 23 and 35 OTUs belong to Bacteroidetes and Firmicutes, respectively. Two OTUs belong to Actinobacteria and two belong to Proteobacteria. In the group fed with CTC, 8 OTUs (7 OTUs belong to Firmicutes) had the highest $(P<0.01)$ normalized relative abundance (NRA), but no OTUs had the lowest NRA among 8 treatments. In the group fed with a mixture of CTC, ZB and CT, 15 OTUs had the lowest $(P<0.01)$ NRA, only one OTUs had the highest $(P<0.01)$ NRA. In the groups fed with a mixture of CTC, OLA and ER, and a mixture of CTC, OLACOV, 7 and 9 OTUs that belong to Bacteroidetes had the highest $(P<0.01)$ NRA, 8 and 12 OTUs that belong to Firmicutes had the lowest $(P<0.01)$ NRA, respectively.

\section{Microbial diversity of the pig fecal microbiota}

Alpha-diversity was used in microbial diversity analysis within-community. The overall average Chaol of all samples, a nonparametric estimator of species richness, was $641.85 \pm 5.97(\mathrm{SEM})$. The Chao1 was less $(P<0.05)$ in the groups fed with a mixture of ZB and CT, a mixture of CTC, OLA and ER, and a mixture of CTC, OLA and VIR than in the control group (Table 4). The average number of OTUs per sample was $597.15 \pm 5.59$ (SEM). The number of observed species became less $(P<0.05)$ in the groups fed with a mixture of ZB and CT, a mixture of CTC, OLA and ER, and a 
mixture of CTC, OLA and VIR, than in the control group (Table 4). The average value for the Shannon and Simpson index, which measures both species richness and abundance, were $6.44 \pm 0.05$ and $0.966 \pm 0.002$, respectively (SEM of the mean).

UPGMA (Unweighted pair-group method with arithmetic mean) UniFrac clustering of antibiotic combination groups was based on weighted unifrac distance matrix, in which pigs fed with a mixture of CTC, OLA and VIR, and a mixture of CTC, OLA and ER are clustered together and differ with other pigs. The same results are shown in the cluster distribution of fecal microbiota of pigs at the level of bacterial genus $(>0.1 \%)$ by heatmap (Fig. S4).

Weighted UniFrac distances were used to as $\beta$-diversity and to compare the eight treatments. The weighted UniFrac distances were less in the groups fed with a mixture of ZB and COL $(P<0.001)$, a mixture of CTC, OLA and ER, and a mixture of CTC, OLA and VIR $(P<0.01)$ than in the control group (Fig. 2a). The principal coordinates analysis (PCoA) plot of the weighted UniFrac distances $(\mathrm{PC} 1=43.33 \%, \mathrm{PC} 2=15.62)$ suggested there was a greater clustering in the groups fed with a mixture of ZB and CT, a mixture of CTC, OLA and ER, and a mixture of CTC, OLA and VIR (Fig. 2b).

\section{Discussion}

The pig gut microbiota is comprised of a large number of diverse microorganisms, such as Bacteria, fungi, Protozoa, viruses and Archaea, which contribute to the animal health (Lamendella et al. 2011; Sommer and Bäckhed 2013). The structure of the gut microbiota depends on some factors, such as diet, age, genetics, and in some cases antimicrobial exposure (Holman and Chénier 2015; Scott 
et al. 2013). Antimicrobial growth promoters cannot be well absorbed in the gut and can affect directly on the gut microbiota (Erik and Knudsen 2001).

In the previous study, Allen et al. (2013) found that combination of CTC, sulfamethazine and penicillin altered the fecal bacterial microbiota and bacteriophage community in the feces of pigs. For example, the relative abundance of genera Coprococcus, Succinivibrio, Streptococcus, Treponema, and Turicibacter was decreased. After continuous subtherapeutic levels of in-feed CTC administration, the relative abundance of Bacteria at 3 weeks of age, the genus Lactobacillus at 6 weeks of age and the phylum Firmicutes at 19 weeks of age were decreased (Holman and Chenier 2014), while the relative abundance of phylum Firmicutes was increased and Proteobacteria was decreased at 9 weeks of age in the present study.

In addition, Holman and Chenier (2014) found that in-feed tylosin increased the relative abundance of genera Coprococcus (3 to 9 weeks of age) and Streptococcus (6 weeks of age), and decreased Fibrobacteres (3 weeks of age), Bacteroidetes ( 9 weeks of age), Succinivibrio (9 weeks of age). However, Kim et al. (2012) showed that tylosin (44 $\mathrm{mg} \mathrm{kg}^{-1}$ feed) administration decreased the relative abundance of the genera Prevotella, Megasphaera, Blautia, Sarcina but increased Lactobacillus, Sporacetigenium, Acetanaerobacterium, and Eggerthella. The results of these two studies are lacking good consistency, which suggests that continuous subtherapeutic antibiotic administration is not the determinant of intestinal microflora diversity.

In China, CTC, B, CT, OLA, ER, and VIR are widely used in pig production. Therefore, the effects of these antibiotic combinations on the abundance and diversity 
intestinal microflora have important significance for the pig production. In the present study, the $\alpha$-diversity indexes Chao 1 and observed species of groups fed with a mixture of ZB and COL, a mixture of CTC, OLA and ER, and a mixture of CTC, OLA and VIR was lower than that of control group, which means that the mixture of ZB and CT, the mixture of CTC, OLA and ER, and the mixture of CTC, OLA and VIR reduced the diversity of fecal microbes. The Shannon index ranged from 6.18-6.53, similar to the values of previously studies (Holman and Chenier 2014; Kim et al. 2012).

Weighted UniFrac is a $\beta$-diversity measure that uses phylogenetic information to compare environmental samples. Weighted UniFrac, coupled with standard multivariate statistical techniques including PCoA, identifies factors explaining differences between microbial communities (Lozupone et al. 2011). A smaller weighted UniFrac value indicates lower the microbial diversity within the group. In the present study, weighted UniFrac distances were less in the groups fed with a mixture of ZB and CT, a mixture of CTC, OLA and ER, and a mixture of CTC, OLA and VIR than in the control group, which is consistent with the $\alpha$-diversity indexes. The PCoA plot of the weighted UniFrac distances had greater clustering in the groups fed with a mixture of ZB and CT, a mixture of CTC, OLA and ER, and a mixture of CTC, OLA and VIR. The results indicate that the combination ZB and CT, CTC combination with OLA and ER or CTC combination with OLA and VIR reduced the diversity of fecal microbes within the group. Previous studies found that B, ER and VIR are microbicide with activity against Gram-positive bacteria (Abudabos 2012; 
Kawakami et al. 1971); COL and OLA are microbicide with activity against Gram-negative bacteria (Guyonnet et al. 2010; Jeong et al. 2009). These suggest that combination of antimicrobial growth promoters with different antibacterial mechanisms should have a better antimicrobial effect.

In the present study, the large majority of sequences were classified as either Firmicutes or Bacteroidetes, which are in agreement with several other studies (Holman and Chenier 2014; Looft et al. 2014b; Looft et al. 2012). Similarly, previous studies showed that Prevotella was the most abundant genus (Holman and Chenier 2014; Looft et al. 2014a), which is in agreement with our study. In our present study, the relative abundance of the phylum Bacteroidetes was increased in the COE and COV groups, and the genus Prevotella, which belongs to the phylum Bacteroidetes (Leser et al. 2002), was increased in the COE and COV groups. Our results showed that CTC combination with OLA and ER, or CTC combination with OLA and VIR, increased the relative abundance of the genus Prevotella. Interestingly, Ley et al. (2006) found that the relative proportion of the phylum Bacteroidetes was decreased in the obese people, which suggest that Bacteroidetes is connected with growth performance. But in our present study, the increase of Bacteroidetes did not increase the growth performance of pigs (Results not presented).

In the present study, the microbial genomic DNA extraction was performed using a bead-beating and CTAB method, which was used by the previous studies ( $\mathrm{Li}$ et al. 2016; Simpson et al. 1999; Wang et al. 2007). But the DNA-isolation protocol of samples influences the distribution of fecal microbes (Knudsen et al. 2016), which 
means that some phyla may be underrepresented simply due to bias of the isolation protocol.

\section{Conclusions}

Understanding the mechanism of how AGPs regulate gut microbiota of animals will aid in the research and development of alternatives to antibiotics. In the present study, the effects of subtherapeutic antibiotic combinations on the relative abundance and diversity of fecal microorganisms were complicated. At the phylum level, the relative abundance of Firmicutes, Bacteroidetes, and Proteobacteria has differences between antibiotic combination treatments. The mixture of CTC, OLA and ER, and the mixture of CTC, OLA and VIR reduced the microbial diversity in the feces of pigs, in which the relative abundance of the phylum Bacteroidetes and the genus Prevotella were increased. Compared with the pigs without antibiotics, the relative abundance of the phymum Proteobacteria and the genus Succinivibrio were decreased except in the pigs fed combination of CTC and Macleaya cordata, and a mixture of CTC, OLA and VIR. Since the effects of antibiotic combinations are complicated, we should not only consider additive effects but also the interaction effects between antibiotics.

\section{Acknowledgments}

We thank Jiangsu Yong-kang Agricultural and Animal Husbandry Co.Ltd. for the experimental animals.

\section{References}


Abudabos, A.M. 2012. Effect of Primalac (R) or Enramycin supplementation on performance, intestinal morphology and microbiology of broilers under Clostridium perfringens challenge. J Food Agric Environ 10(3-4): 595-599.

Ahmed, A., Azim, A., Gurjar, M., and Baronia, A.K. 2014. Current concepts in combination antibiotic therapy for critically ill patients. Indian J Crit Care Med 18(5): 310-314. doi: 10.4103/0972-5229.132495. PMID: 4047693.

Allen, H.K., Levine, U.Y., Looft, T., Bandrick, M., and Casey, T.A. 2013. Treatment, promotion, commotion: antibiotic alternatives in food-producing animals. Trends in microbiology 21(3): 114-119. doi: 10.1016/j.tim.2012.11.001.

Caporaso, J.G., Lauber, C.L., Walters, W.A., Berg-Lyons, D., Lozupone, C.A., Turnbaugh, P.J., Fierer, N., and Knight, R. 2011. Global patterns of 16S rRNA diversity at a depth of millions of sequences per sample. Proc Natl Acad Sci U S A 108 Suppl 1: 4516-4522. doi: 10.1073/pnas.1000080107. PMID: 3063599 .

Castillo, M., Martin-Orue, S.M., Roca, M., Manzanilla, E.G., Badiola, I., Perez, J.F., and Gasa, J. 2006. The response of gastrointestinal microbiota to avilamycin, butyrate, and plant extracts in early-weaned pigs. Journal of animal science 84(10): 2725-2734. doi: 10.2527/jas.2004-556.

Chen, Q., Tang, S., Jin, X., Zou, J., Chen, K., Zhang, T., and Xiao, X. 2009. Investigation of the genotoxicity of quinocetone, carbadox and olaquindox in vitro using Vero cells. Food Chem Toxicol 47(2): 328-334.

Chopra, I., and Roberts, M. 2001. Tetracycline antibiotics: mode of action, applications, molecular biology, and epidemiology of bacterial resistance. Microbiology and molecular biology reviews : MMBR 65(2): 232-260. doi: 10.1128/MMBR.65.2.232-260.2001. PMID: 99026. 
Cromwell, G.L. 2002. Why and how antibiotics are used in swine production. Animal biotechnology 13(1): 7-27. doi: 10.1081/ABIO-120005767.

Engberg, R.M., Hedemann, M.S., Leser, T.D., and Jensen, B.B. 2000. Effect of zinc bacitracin and salinomycin on intestinal microflora and performance of broilers. Poultry science 79(9): 1311-1319.

Erik, K., and Knudsen, B. 2001. Development of antibiotic resistance and options to replace antimicrobials in animal diets. Proceedings of the nutrition society 60(03): 291-299.

Gong, J., Yu, H., Liu, T., Gill, J.J., Chambers, J.R., Wheatcroft, R., and Sabour, P.M. 2008. Effects of zinc bacitracin, bird age and access to range on bacterial microbiota in the ileum and caeca of broiler chickens. Journal of applied microbiology 104(5): 1372-1382. doi: 10.1111/j.1365-2672.2007.03699.x.

Guyonnet, J., Manco, B., Baduel, L., Kaltsatos, V., Aliabadi, M.H., and Lees, P. 2010. Determination of a dosage regimen of colistin by pharmacokinetic/pharmacodynamic integration and modeling for treatment of G.I.T. disease in pigs. Res Vet Sci 88(2): 307-314. doi: 10.1016/j.rvsc.2009.09.001.

Holman, D.B., and Chénier, M.R. 2015. Antimicrobial use in swine production and its effect on the swine gut microbiota and antimicrobial resistance. Canadian journal of microbiology 61(11): 785-798.

Holman, D.B., and Chenier, M.R. 2014. Temporal changes and the effect of subtherapeutic concentrations of antibiotics in the gut microbiota of swine. FEMS microbiology ecology 90(3): 599-608. doi: 10.1111/1574-6941.12419.

Jeong, S.H., Song, Y.K., and Cho, J.H. 2009. Risk assessment of ciprofloxacin, flavomycin, olaquindox and colistin sulfate based on microbiological impact on human gut biota. Regulatory toxicology and pharmacology : RTP 53(3): 209-216. doi: 10.1016/j.yrtph.2009.01.004.

Johnson, B.A., Anker, H., and Meleney, F.L. 1945. Bacitracin: A New Antibiotic Produced by a Member of the B. Subtilis Group. Science 102(2650): 376-377. doi: 10.1126/science.102.2650.376. 
Kawakami, M., Nagai, Y., Fujii, T., and Mitsuhashi, S. 1971. Anti-microbial activities of enduracidin (enramycin) in vitro and in vivo. The Journal of antibiotics 24(9): 583-586.

Kim, H.B., Borewicz, K., White, B.A., Singer, R.S., Sreevatsan, S., Tu, Z.J., and Isaacson, R.E. 2012. Microbial shifts in the swine distal gut in response to the treatment with antimicrobial growth promoter, tylosin. Proc Natl Acad Sci U S A 109(38): 15485-15490. doi: 10.1073/pnas.1205147109. PMID: 3458334.

Knudsen, B.E., Bergmark, L., Munk, P., Lukjancenko, O., Prieme, A., Aarestrup, F.M., and Pamp, S.J. 2016. Impact of Sample Type and DNA Isolation Procedure on Genomic Inference of Microbiome Composition. mSystems 1(5). doi: 10.1128/mSystems.00095-16. PMID: 5080404.

Kosina, P., Gregorova, J., Gruz, J., Vacek, J., Kolar, M., Vogel, M., Roos, W., Naumann, K., Simanek, V., and Ulrichova, J. 2010. Phytochemical and antimicrobial characterization of Macleaya cordata herb. Fitoterapia 81(8): 1006-1012. doi: 10.1016/j.fitote.2010.06.020.

Lamendella, R., Domingo, J.W., Ghosh, S., Martinson, J., and Oerther, D.B. 2011. Comparative fecal metagenomics unveils unique functional capacity of the swine gut. BMC microbiology 11: 103. doi: 10.1186/1471-2180-11-103. PMID: 3123192.

Leser, T.D., Amenuvor, J.Z., Jensen, T.K., Lindecrona, R.H., Boye, M., and Moller, K. 2002. Culture-independent analysis of gut bacteria: the pig gastrointestinal tract microbiota revisited. Applied and environmental microbiology 68(2): 673-690. PMID: 126712.

Ley, R.E., Turnbaugh, P.J., Klein, S., and Gordon, J.I. 2006. Microbial ecology: human gut microbes associated with obesity. Nature 444(7122): 1022-1023. doi: 10.1038/4441022a.

Li, H., Chu, Q., Xu, F., Fu, L., Liang, T., Li, Y., and Zhou, B. 2016. Combination of antibiotics suppressed the increase of a part of ARGs in fecal microorganism of weaned pigs. Environmental 
science and pollution research international 23(18): 18183-18191. doi: 10.1007/s11356-016-7004-7.

Looft, T., Allen, H.K., Cantarel, B.L., Levine, U.Y., Bayles, D.O., Alt, D.P., Henrissat, B., and Stanton, T.B. 2014a. Bacteria, phages and pigs: the effects of in-feed antibiotics on the microbiome at different gut locations. The ISME journal 8(8): 1566-1576. doi: 10.1038/ismej.2014.12. PMID: 4817603.

Looft, T., Allen, H.K., Casey, T.A., Alt, D.P., and Stanton, T.B. 2014b. Carbadox has both temporary and lasting effects on the swine gut microbiota. Frontiers in microbiology 5: 276. doi: 10.3389/fmicb.2014.00276. PMID: 4050737.

Looft, T., Johnson, T.A., Allen, H.K., Bayles, D.O., Alt, D.P., Stedtfeld, R.D., Sul, W.J., Stedtfeld, T.M., Chai, B., Cole, J.R., Hashsham, S.A., Tiedje, J.M., and Stanton, T.B. 2012. In-feed antibiotic effects on the swine intestinal microbiome. Proc Natl Acad Sci U S A 109(5): 1691-1696. doi: 10.1073/pnas.1120238109. PMID: 3277147.

Lozupone, C., Lladser, M.E., Knights, D., Stombaugh, J., and Knight, R. 2011. UniFrac: an effective distance metric for microbial community comparison. The ISME journal 5(2): 169-172. doi: 10.1038/ismej.2010.133. PMID: 3105689.

Nakajima, K., and Kawamata, J. 1965. Studies on the mechanism of action of colistin. II. Alteration of permeability of Escherichia coli by colistin. Biken J 8(4): 233-239.

Rettedal, E., Vilain, S., Lindblom, S., Lehnert, K., Scofield, C., George, S., Clay, S., Kaushik, R.S., Rosa, A.J., Francis, D., and Brozel, V.S. 2009. Alteration of the ileal microbiota of weanling piglets by the growth-promoting antibiotic chlortetracycline. Applied and environmental microbiology 75(17): 5489-5495. doi: 10.1128/AEM.02220-08. PMID: 2737922.

Scott, K.P., Gratz, S.W., Sheridan, P.O., Flint, H.J., and Duncan, S.H. 2013. The influence of diet on the gut microbiota. Pharmacological research : the official journal of the Italian Pharmacological Society 
69(1): 52-60. doi: 10.1016/j.phrs.2012.10.020.

Simpson, J.M., McCracken, V.J., White, B.A., Gaskins, H.R., and Mackie, R.I. 1999. Application of denaturant gradient gel electrophoresis for the analysis of the porcine gastrointestinal microbiota. Journal of microbiological methods 36(3): 167-179.

Sommer, F., and Bäckhed, F. 2013. The gut microbiota—masters of host development and physiology. Nature Reviews Microbiology 11(4): 227-238.

Stewart, L.L., Kim, B.G., Gramm, B.R., Nimmo, R.D., and Stein, H.H. 2010. Effect of virginiamycin on the apparent ileal digestibility of amino acids by growing pigs. Journal of animal science $\mathbf{8 8}(5)$ : 1718-1724. doi: 10.2527/jas.2009-2063.

van der Fels-Klerx, H.J., Puister-Jansen, L.F., van Asselt, E.D., and Burgers, S.L. 2011. Farm factors associated with the use of antibiotics in pig production. Journal of animal science 89(6): 1922-1929. doi: $10.2527 /$ jas.2010-3046.

Wang, H.F., Zhu, W.Y., Yao, W., and Liu, J.X. 2007. DGGE and 16S rDNA sequencing analysis of bacterial communities in colon content and feces of pigs fed whole crop rice. Anaerobe 13(3-4): 127-133.

Yatsunenko, T., Rey, F.E., Manary, M.J., Trehan, I., Dominguez-Bello, M.G., Contreras, M., Magris, M., Hidalgo, G., Baldassano, R.N., Anokhin, A.P., Heath, A.C., Warner, B., Reeder, J., Kuczynski, J., Caporaso, J.G., Lozupone, C.A., Lauber, C., Clemente, J.C., Knights, D., Knight, R., and Gordon, J.I. 2012. Human gut microbiome viewed across age and geography. Nature 486(7402): 222-227. doi: 10.1038/nature11053. PMID: 3376388.

Youssef, N., Sheik, C.S., Krumholz, L.R., Najar, F.Z., Roe, B.A., and Elshahed, M.S. 2009. Comparison of species richness estimates obtained using nearly complete fragments and simulated 
pyrosequencing-generated fragments in 16S rRNA gene-based environmental surveys. Applied and environmental microbiology 75(16): 5227-5236. doi: 10.1128/AEM.00592-09. PMID: 2725448. 


\section{Figure Legends}

Fig. 1. The relative abundances of top 10 phylum (a) and genus (b) in the eight groups $(\mathrm{n}=6)$

CT: Control group; CTC: Chlortetracycline; BC: bacitracin zinc and colistin sulphate; CBC: chlortetracycline, bacitracin zinc and colistin sulphate; CS: chlortetracycline and Macleaya cordata extracts; $\mathrm{CO}$ : chlortetracycline and olaquindox; $\mathrm{COE}$ :

chlortetracycline, olaquindox and enramycin; COV: chlortetracycline, olaquindox and virginiamycin. 
Fig. 2. Weighted Unifrac distances in the eight groups (a) and PCoA of the weighted UniFrac distances (b). Principal components (PCs) 1 and 2 explained $43.3 \%$ and $15.6 \%$ of the variance, respectively.

CT: Control group; CTC: Chlortetracycline; BC: bacitracin zinc and colistin sulphate; CBC: chlortetracycline, bacitracin zinc and colistin sulphate; $\mathrm{CO}$ : chlortetracycline and olaquindox; CS: chlortetracycline and Macleaya cordata extracts; COE:

chlortetracycline, olaquindox and enramycin; COV: chlortetracycline, olaquindox and virginiamycin. 
Table 1. Treatments for in-feed antibiotic additives for weaned pigs.

\begin{tabular}{lll}
\hline Groups & Weaned pigs & Antibiotics additives \\
\hline CT & 50 & Fed basal diet (without antibiotics) \\
CTC & 50 & $75 \mathrm{mg} / \mathrm{kg}$ chlortetracycline (CTC) (feed grade premixed agent) \\
& & $40 \mathrm{mg} / \mathrm{kg}$ zinc bacitracin (ZB) and $20 \mathrm{mg} / \mathrm{kg}$ colistin sulphate \\
BC & 50 & $(\mathrm{COL})$ \\
CBC & 50 & $75 \mathrm{mg} / \mathrm{kg} \mathrm{CTC}, 40 \mathrm{mg} / \mathrm{kg} \mathrm{ZB}$ and $20 \mathrm{mg} / \mathrm{kg}$ CT \\
CS & 50 & $75 \mathrm{mg} / \mathrm{kg} \mathrm{CTC}$ and $50 \mathrm{mg} / \mathrm{kg}$ Macleaya cordata extracts \\
CO & 50 & $75 \mathrm{mg} / \mathrm{kg} \mathrm{CTC}$ and $100 \mathrm{mg} / \mathrm{kg}$ olaquindox (OLA) \\
COE & 50 & $75 \mathrm{mg} / \mathrm{kg} \mathrm{CTC}, 100 \mathrm{mg} / \mathrm{kg}$ OLA and $20 \mathrm{mg} / \mathrm{kg}$ enramycin (ER) \\
& & $75 \mathrm{mg} / \mathrm{kg} \mathrm{CTC}, 100 \mathrm{mg} / \mathrm{kg}$ OLA and $15 \mathrm{mg} / \mathrm{kg}$ virginiamycin \\
COV & 50 & $(\mathrm{VIR})$ \\
\hline
\end{tabular}


Table 2. Comparison of the proportions of dominant phyla in the eight groups of pigs fed different antibiotic combinations for 28 days.

\begin{tabular}{llll}
\hline Groups & Firmicutes & Bacteroidetes & Proteobacteria \\
\hline CT & $0.518 \pm 0.032$ & $0.381 \pm 0.006$ & $0.076 \pm 0.031$ \\
CTC & $0.594 \pm 0.016^{*}$ & $0.352 \pm 0.013$ & $0.027 \pm 0.007^{*}$ \\
BC & $0.604 \pm 0.011^{*}$ & $0.369 \pm 0.011$ & $0.018 \pm 0.003^{* *}$ \\
CBC & $0.571 \pm 0.025$ & $0.389 \pm 0.022$ & $0.026 \pm 0.009^{*}$ \\
CS & $0.560 \pm 0.019$ & $0.385 \pm 0.017$ & $0.039 \pm 0.014$ \\
CO & $0.577 \pm 0.039$ & $0.365 \pm 0.023$ & $0.030 \pm 0.014^{*}$ \\
COE & $0.470 \pm 0.025$ & $0.497 \pm 0.023^{* *}$ & $0.019 \pm 0.003^{* *}$ \\
COV & $0.437 \pm 0.010^{*}$ & $0.496 \pm 0.008^{* *}$ & $0.051 \pm 0.015$ \\
\hline
\end{tabular}

Note: CT: Fed basal diet (without antibiotics); CTC: $75 \mathrm{mg} / \mathrm{kg}$ chlortetracycline (CTC) (feed grade premixed agent); BC: $40 \mathrm{mg} / \mathrm{kg}$ zinc bacitracin (ZB) and $20 \mathrm{mg} / \mathrm{kg}$ colistin sulphate (COL);CBC: $75 \mathrm{mg} / \mathrm{kg}$ CTC, $40 \mathrm{mg} / \mathrm{kg} \mathrm{ZB}$ and 20mg/kg CT; CS: 75 $\mathrm{mg} / \mathrm{kg} \mathrm{CTC}$ and $50 \mathrm{mg} / \mathrm{kg}$ Macleaya cordata extracts; CO: $75 \mathrm{mg} / \mathrm{kg}$ CTC and 100mg/kg olaquindox (OLA);COE: $75 \mathrm{mg} / \mathrm{kg} \mathrm{CTC,} 100 \mathrm{mg} / \mathrm{kg}$ OLA and 20mg/kg enramycin (ER);COV: 75 mg/kg CTC, $100 \mathrm{mg} / \mathrm{kg}$ OLA and 15mg/kg virginiamycin (VIR). The data are presented as mean \pm SEM. Compared with the CT group: ${ }^{*} P<$ $0.05 ; * * P<0.01$ 
Table 3. Comparison of dominant genus in the eight groups.

\begin{tabular}{lllll}
\hline Groups & Prevotella & Roseburia & Succinivibrio & Faecalibacterium \\
\hline CT & $0.158 \pm 0.014$ & $0.061 \pm 0.010$ & $0.071 \pm 0.032$ & $0.047 \pm 0.012$ \\
CTC & $0.123 \pm 0.017$ & $0.116 \pm 0.031$ & $0.023 \pm 0.007^{*}$ & $0.036 \pm 0.004$ \\
BC & $0.151 \pm 0.015$ & $0.107 \pm 0.009$ & $0.013 \pm 0.003^{* *}$ & $0.060 \pm 0.011$ \\
CBC & $0.160 \pm 0.029$ & $0.085 \pm 0.036$ & $0.022 \pm 0.009^{*}$ & $0.038 \pm 0.004$ \\
CS & $0.181 \pm 0.026$ & $0.090 \pm 0.010$ & $0.034 \pm 0.014$ & $0.056 \pm 0.005$ \\
CO & $0.145 \pm 0.011$ & $0.068 \pm 0.022$ & $0.027 \pm 0.013^{*}$ & $0.039 \pm 0.008$ \\
COE & $0.307 \pm 0.021^{* *}$ & $0.080 \pm 0.017$ & $0.016 \pm 0.003^{*}$ & $0.019 \pm 0.003^{* *}$ \\
COV & $0.286 \pm 0.031^{* *}$ & $0.069 \pm 0.013$ & $0.048 \pm 0.015$ & $0.033 \pm 0.007$ \\
\hline
\end{tabular}

Note: CT: Fed basal diet (without antibiotics); CTC: $75 \mathrm{mg} / \mathrm{kg}$ chlortetracycline (CTC) (feed grade premixed agent); BC: $40 \mathrm{mg} / \mathrm{kg}$ zinc bacitracin (ZB) and $20 \mathrm{mg} / \mathrm{kg}$ colistin sulphate (COL);CBC: $75 \mathrm{mg} / \mathrm{kg}$ CTC, $40 \mathrm{mg} / \mathrm{kg}$ ZB and 20mg/kg CT; CS: 75 $\mathrm{mg} / \mathrm{kg} \mathrm{CTC}$ and $50 \mathrm{mg} / \mathrm{kg}$ Macleaya cordata extracts; CO: $75 \mathrm{mg} / \mathrm{kg} \mathrm{CTC}$ and 100mg/kg olaquindox (OLA);COE: $75 \mathrm{mg} / \mathrm{kg} \mathrm{CTC,} 100 \mathrm{mg} / \mathrm{kg}$ OLA and 20mg/kg enramycin (ER);COV: $75 \mathrm{mg} / \mathrm{kg}$ CTC, $100 \mathrm{mg} / \mathrm{kg}$ OLA and 15mg/kg virginiamycin (VIR). The data are presented as mean \pm SEM. Compared with the CT group: ${ }^{*} P<$ $0.05 ; * * P<0.01$ 
Table 4. Comparison of Alpha diversity index in the eight groups.

\begin{tabular}{llllll}
\hline Groups & observed_species & shannon & simpson & chao1 & goods_coverage \\
\hline CT & $624.83 \pm 17.01$ & $6.52 \pm 0.19$ & $0.967 \pm 0.0078$ & $673.16 \pm 16.2$ & $0.9980 \pm 0.00$ \\
CTC & $609.17 \pm 13.47$ & $6.48 \pm 0.18$ & $0.965 \pm 0.0083$ & $650.70 \pm 13.7$ & $0.9980 \pm 0.00$ \\
BC & $583.17 \pm 12.61^{*}$ & $6.49 \pm 0.14$ & $0.973 \pm 0.0045$ & $625.75 \pm 12.7^{*}$ & $0.9980 \pm 0.00$ \\
CBC & $613.00 \pm 22.71$ & $6.18 \pm 0.16$ & $0.952 \pm 0.0080$ & $663.05 \pm 24.8$ & $0.9978 \pm 0.00$ \\
CS & $603.67 \pm 9.96$ & $6.38 \pm 0.14$ & $0.968 \pm 0.0056$ & $649.68 \pm 9.8$ & $0.9980 \pm 0.00$ \\
CO & $606.00 \pm 11.12$ & $6.49 \pm 0.16$ & $0.967 \pm 0.0076$ & $653.02 \pm 11.5$ & $0.9980 \pm 0.00$ \\
COE & $557.50 \pm 9.71^{* *}$ & $6.41 \pm 0.13$ & $0.969 \pm 0.0042$ & $603.10 \pm 9.4^{* *}$ & $0.9983 \pm 0.00$ \\
COV & $579.83 \pm 14.62^{*}$ & $6.53 \pm 0.13$ & $0.971 \pm 0.0040$ & $616.37 \pm 17.7 *$ & $0.9983 \pm 0.00$ \\
\hline
\end{tabular}

Note: CT: Fed basal diet (without antibiotics); CTC: $75 \mathrm{mg} / \mathrm{kg}$ chlortetracycline (CTC) (feed grade premixed agent); BC: $40 \mathrm{mg} / \mathrm{kg}$ zinc bacitracin (ZB) and $20 \mathrm{mg} / \mathrm{kg}$ colistin sulphate (COL);CBC: $75 \mathrm{mg} / \mathrm{kg} \mathrm{CTC,} 40 \mathrm{mg} / \mathrm{kg} \mathrm{ZB}$ and 20mg/kg CT; CS: 75 $\mathrm{mg} / \mathrm{kg} \mathrm{CTC}$ and $50 \mathrm{mg} / \mathrm{kg}$ Macleaya cordata extracts; CO: $75 \mathrm{mg} / \mathrm{kg}$ CTC and 100mg/kg olaquindox (OLA);COE: $75 \mathrm{mg} / \mathrm{kg} \mathrm{CTC,} 100 \mathrm{mg} / \mathrm{kg}$ OLA and 20mg/kg enramycin (ER);COV: 75 mg/kg CTC, $100 \mathrm{mg} / \mathrm{kg}$ OLA and 15mg/kg virginiamycin (VIR). The data are presented as mean \pm SEM. Compared with the CT group: ${ }^{*} P<$ $0.05 ; * * P<0.01$ 


\section{Supporting Information}

Fig. S1. The rarefaction curves of treatments in antibiotic combinations.

Fig. S2. OTU-level composition (the relative abundance $>1 \%$ ) of fecal samples in antibiotic combination groups.

Fig. S3. OTUs with significant difference at $1 \%$ level $(\mathrm{P}<0.01)$ between antibiotic combination treatments.

Fig. S4. Cluster distribution of fecal microbiota of pigs at the level of bacterial genus $(>0.1 \%)$ by heatmap.

Table S1. Raw reads, Effective Tags and OTUs from each groups.

Table S2. OTUs with significant difference between different antibiotics combinations.

Table S3. Effect of each group on the relative proportion (percentage \pm standard deviation of the mean) of the most abundant phyla and genera in the each treatment pigs $(\mathrm{n}=6)$.

Table S4. OTU Taxonomy. 

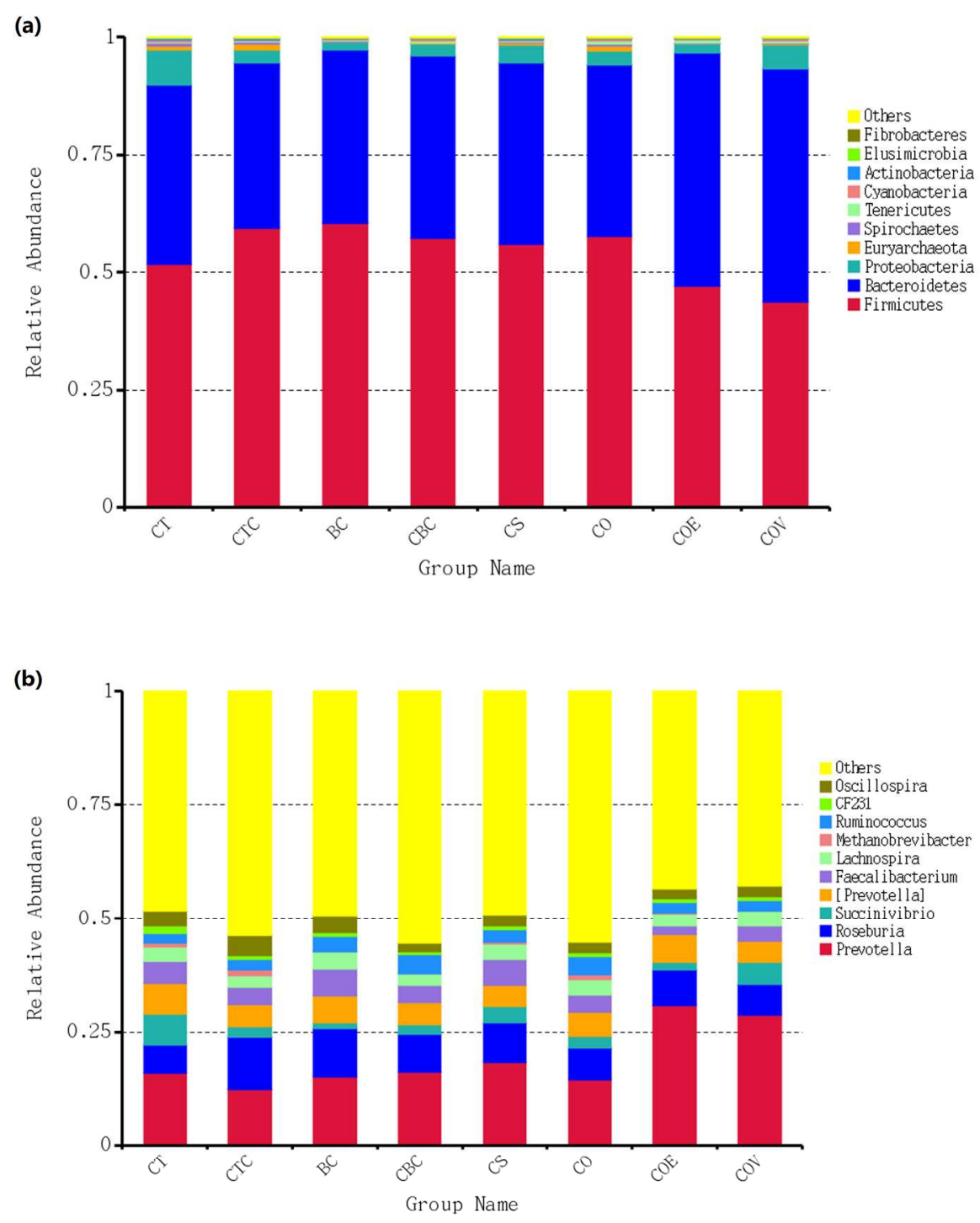

Fig. 1. The relative abundances of top 10 phylum (a) and genus (b) in the eight groups $(n=6)+C T: C o n t r o l$ group; CTC: Chlortetracycline; BC: bacitracin zinc and colistin sulphate; CBC: chlortetracycline, bacitracin zinc and colistin sulphate; CS: chlortetracycline and Macleaya cordata extracts; CO: chlortetracycline and olaquindox; COE: chlortetracycline, olaquindox and enramycin; COV: chlortetracycline, olaquindox and virginiamycin.

Fig. 1

$191 \times 243 \mathrm{~mm}(300 \times 300$ DPI $)$ 

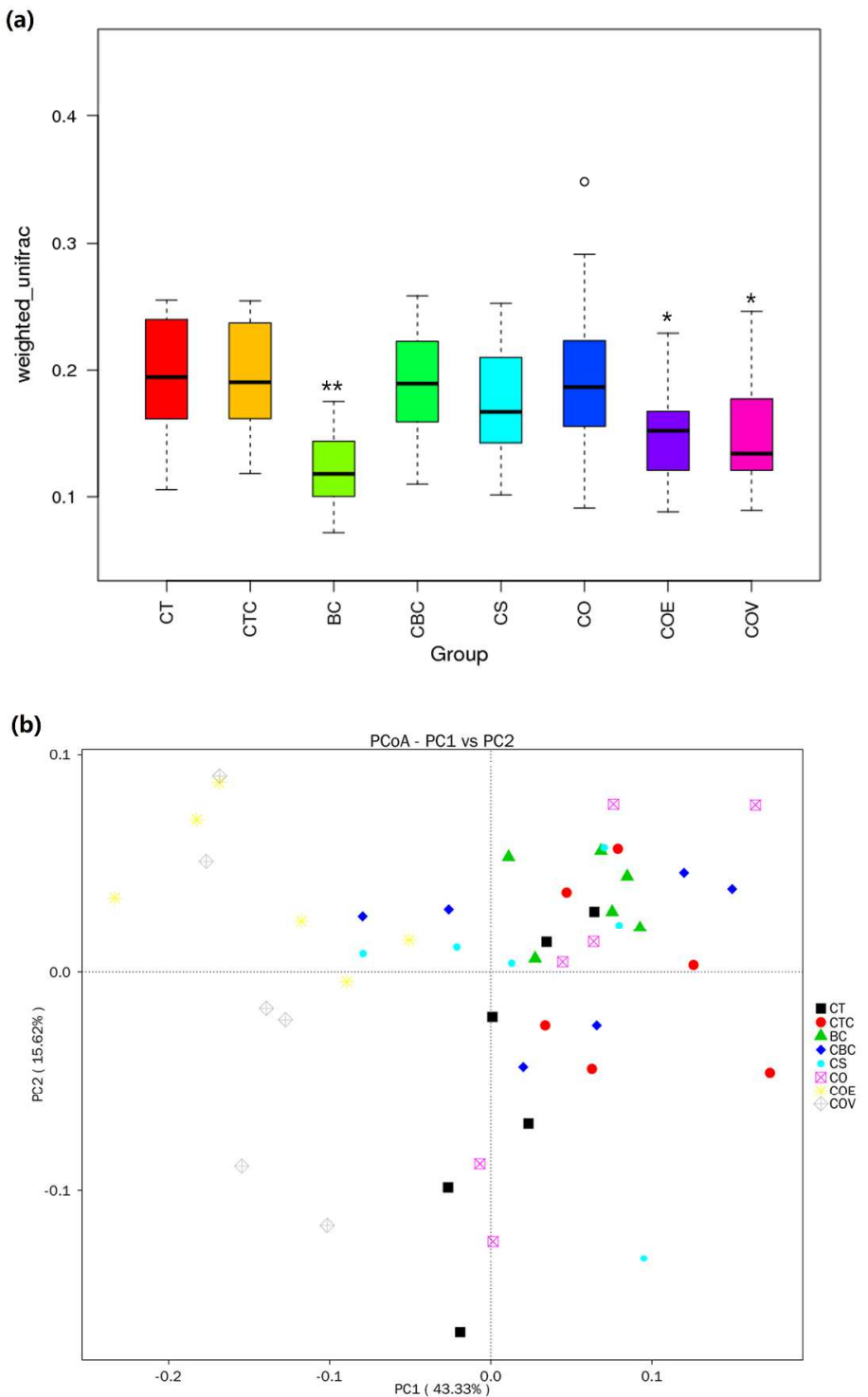

Fig. 2. Weighted Unifrac distances in the eight groups (a) and PCOA of the weighted UniFrac distances (b). Principal components (PCs) 1 and 2 explained $43.3 \%$ and $15.6 \%$ of the variance, respectively.! $+\mathrm{CT}$ : Control group; CTC: Chlortetracycline; BC: bacitracin zinc and colistin sulphate; CBC: chlortetracycline, bacitracin zinc and colistin sulphate; CO: chlortetracycline and olaquindox; CS: chlortetracycline and Macleaya cordata extracts; COE: chlortetracycline, olaquindox and enramycin; COV: chlortetracycline, olaquindox and virginiamycin.

Fig. 2

$260 \times 422 \mathrm{~mm}(300 \times 300$ DPI $)$ 


\section{Supporting Information}

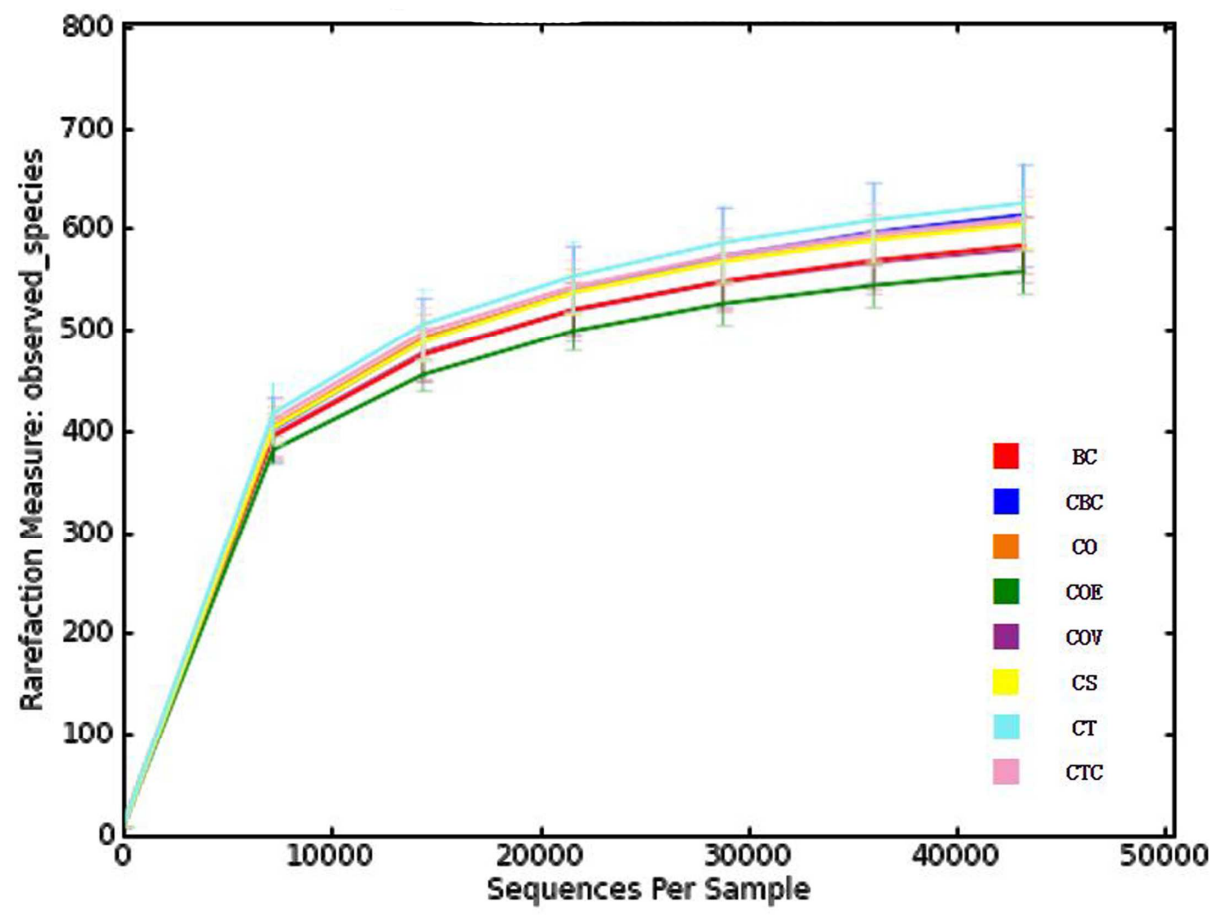

Figure S1 The rarefaction curves of treatments in antibiotic combinations.

CT: Control group $(n=6)$; CTC: Chlortetracycline $(n=6)$; BC: a mixture of bacitracin zinc and colistin sulphate $(n=6)$; CBC: a mixture of chlortetracycline, bacitracin zinc and colistin sulphate $(\mathrm{n}=6)$; CS: a mixture of chlortetracycline and Macleaya cordata extracts ( $\mathrm{n}=6)$; CO: a mixture of chlortetracycline and olaquindox $(n=6)$; COE: a mixture of chlortetracycline, olaquindox and enramycin ( $n=6)$; COV: a mixture of chlortetracycline, olaquindox and virginiamycin $(n=6)$. 


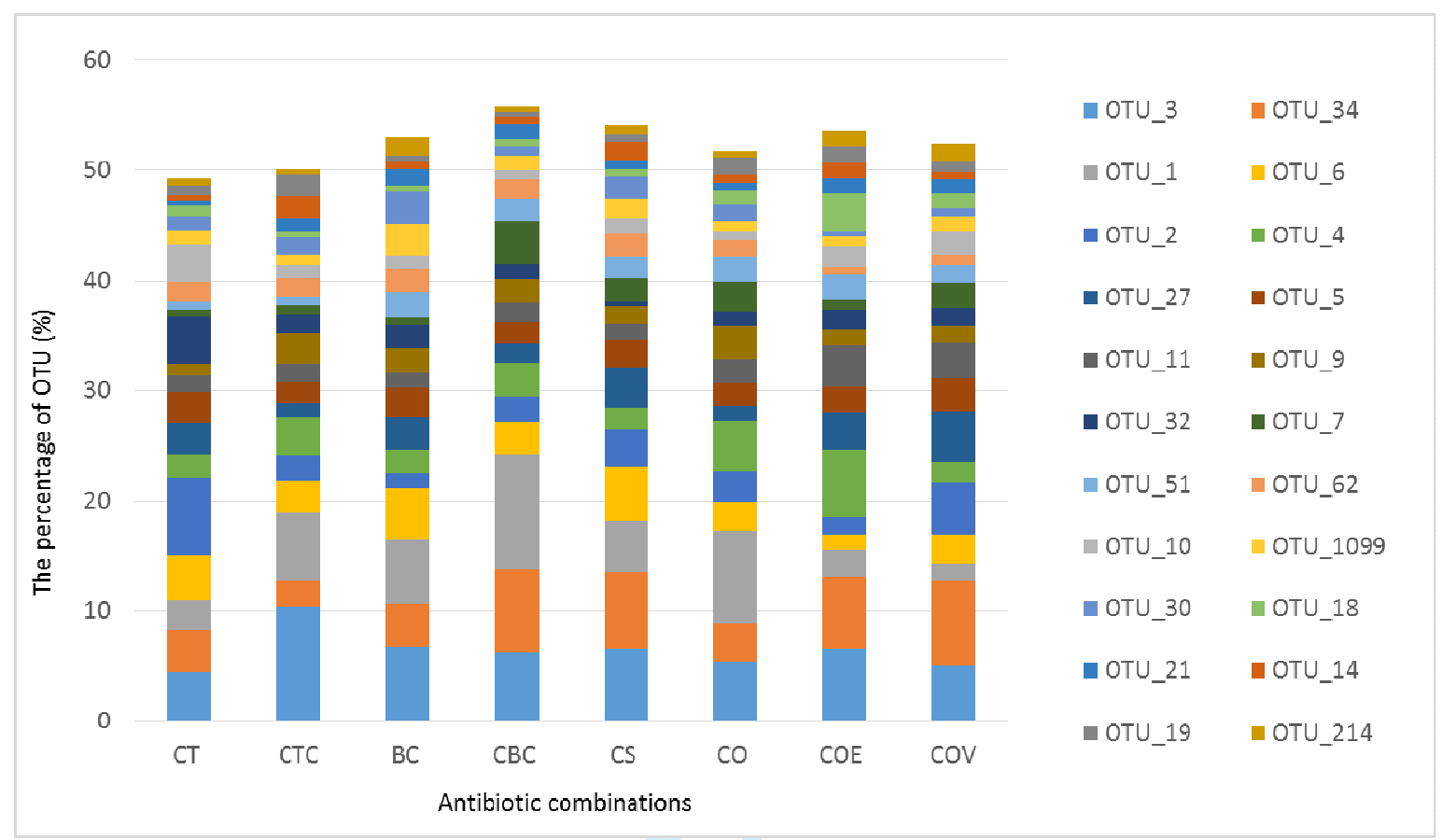

Figure S2 OTU-level composition (the relative abundance $>1 \%$ ) of fecal samples in antibiotic combination groups.

CT: Control group $(n=6)$; CTC: Chlortetracycline $(n=6)$; BC: a mixture of bacitracin zinc and colistin sulphate ( $n=6)$; CBC: a mixture of

chlortetracycline, bacitracin zinc and colistin sulphate $(\mathrm{n}=6)$; CS: a mixture of chlortetracycline and Macleaya cordata extracts ( $\mathrm{n}=6$ ); CO: a mixture of chlortetracycline and olaquindox $(n=6)$; COE: a mixture of chlortetracycline, olaquindox and enramycin $(n=6)$; COV: a mixture of chlortetracycline, olaquindox and virginiamycin $(n=6)$. 


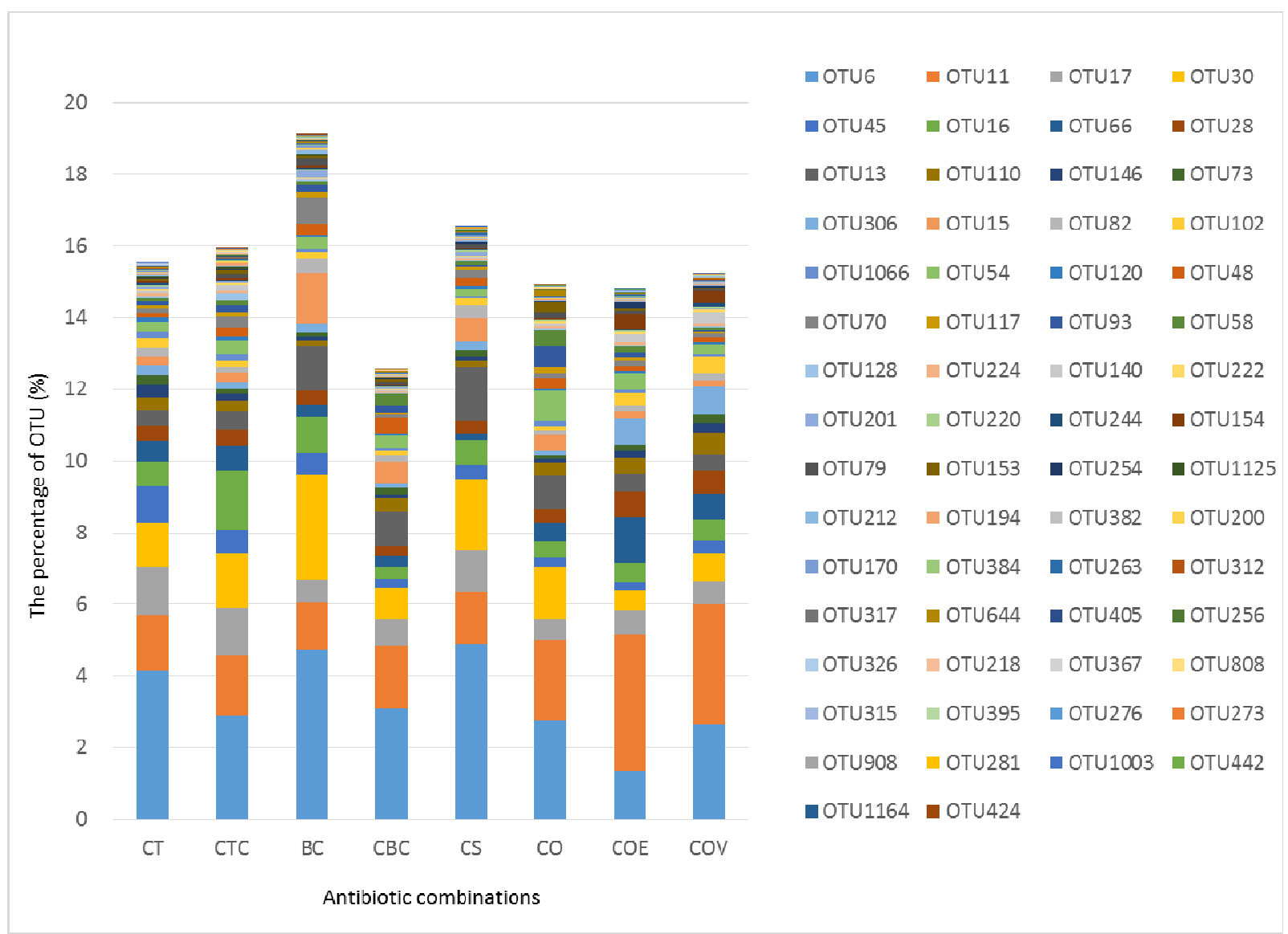

Figure S3 OTUs with significant difference at $1 \%$ level $(P<0.01)$ between antibiotic combination treatments.

CT: Control group $(n=6)$; CTC: Chlortetracycline $(n=6)$; BC: a mixture of bacitracin zinc and colistin sulphate ( $n=6)$; CBC: a mixture of

chlortetracycline, bacitracin zinc and colistin sulphate $(n=6)$; CS: a mixture of chlortetracycline and Macleaya cordata extracts $(n=6)$; CO: a mixture of chlortetracycline and olaquindox $(n=6)$; COE: a mixture of chlortetracycline, olaquindox and enramycin (n=6); COV: a mixture of chlortetracycline, olaquindox and virginiamycin $(n=6)$. 


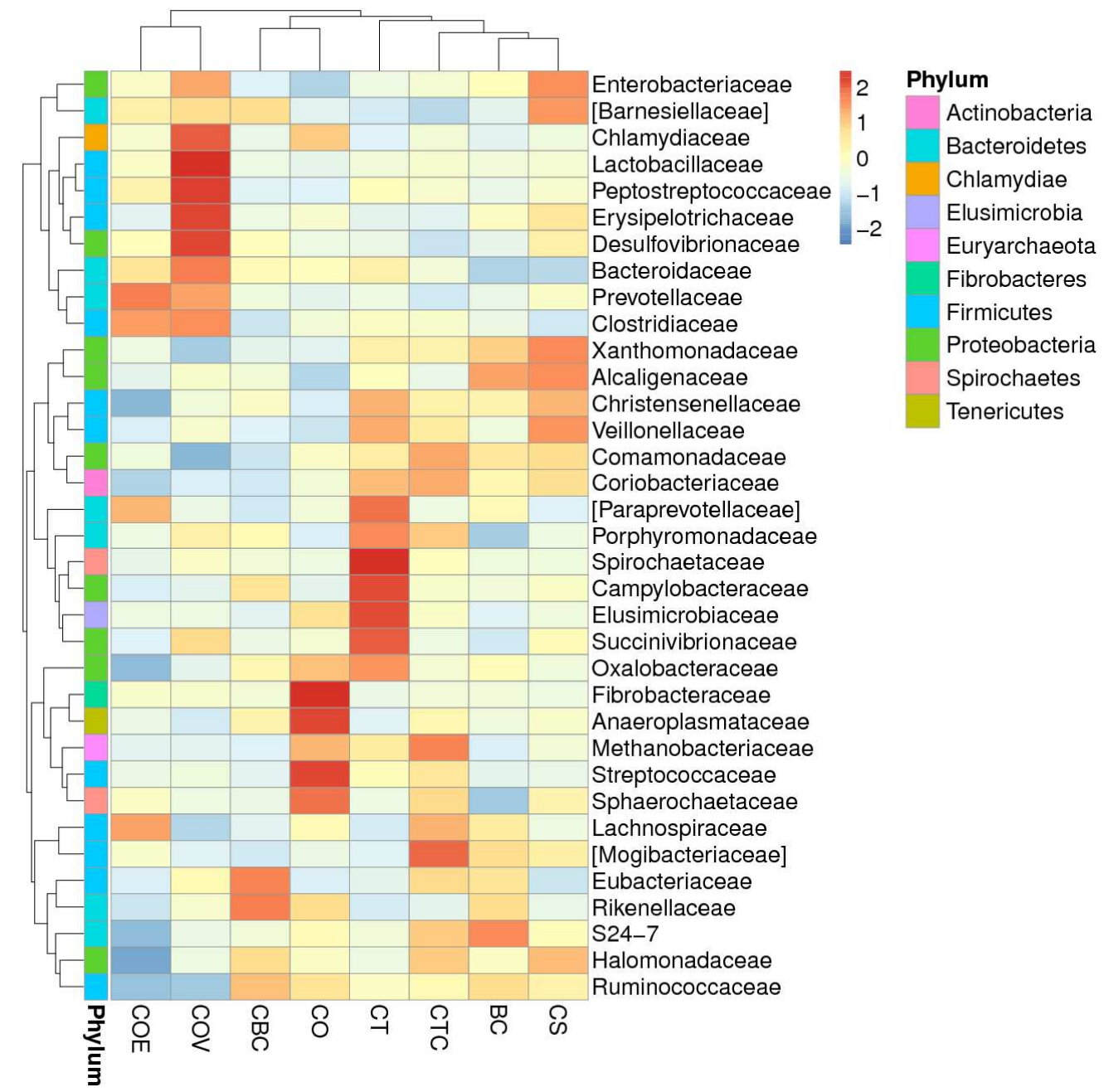

Figure S4 Cluster distribution of fecal microbiota of pigs at the level of bacterial genus ( $>0.1 \%)$ by heatmap.

CT: Control group $(n=6)$; CTC: Chlortetracycline $(n=6)$; BC: a mixture of bacitracin zinc and colistin sulphate ( $n=6)$; CBC: a mixture of

chlortetracycline, bacitracin zinc and colistin sulphate ( $\mathrm{n}=6)$; CS: a mixture of chlortetracycline and Macleaya cordata extracts (n=6); CO: a mixture of chlortetracycline and olaquindox $(n=6)$; COE: a mixture of chlortetracycline, olaquindox and enramycin $(n=6)$; COV: a mixture of chlortetracycline, olaquindox and virginiamycin $(n=6)$. 
Table S2 OTUs with significant difference between different antibiotics combinations

\begin{tabular}{|c|c|c|c|c|c|c|c|c|c|c|c|}
\hline OTU & $\mathrm{P}$ & Microbial group & CT & CTC & $\mathrm{BC}$ & $\mathrm{CBC}$ & $\mathrm{CS}$ & $\mathrm{CO}$ & $\mathrm{COE}$ & $\mathrm{COV}$ & $\begin{array}{c}\mathrm{P} \\
\text { Value }\end{array}$ \\
\hline OTU276 & Actinobacteria & f__Coriobacteriaceae & $0.005 \pm 0.008$ & $0.014 \pm 0.017$ & $0.023 \pm 0.008$ & $0.002 \pm 0.005$ & $0.007 \pm 0.009$ & $0.012 \pm 0.011$ & $0.009 \pm 0.007$ & $0.002 \pm 0.005$ & 0.006 \\
\hline OTU281 & & $\begin{array}{l}\text { f__Coriobacteriaceae; } \\
\text { g__Slackia }\end{array}$ & $0.002 \pm 0.005$ & $0.008 \pm 0.009$ & $0.030 \pm 0.015$ & $0.005 \pm 0.008$ & $0.020 \pm 0.012$ & $0.002 \pm 0.005$ & $0.000 \pm 0.000$ & $0.003 \pm 0.008$ & 0.000 \\
\hline OTU82 & Bacteroidetes & o__Bacteroidales & $0.247 \pm 0.214$ & $0.135 \pm 0.030$ & $0.388 \pm 0.157$ & $0.164 \pm 0.069$ & $0.372 \pm 0.253$ & $0.129 \pm 0.038$ & $0.150 \pm 0.056$ & $0.182 \pm 0.052$ & 0.004 \\
\hline OTU73 & & f__[Paraprevotellaceae & $0.290 \pm 0.172$ & $0.126 \pm 0.023$ & $0.108 \pm 0.028$ & $0.174 \pm 0.071$ & $0.150 \pm 0.029$ & $0.092 \pm 0.020$ & $0.156 \pm 0.021$ & $0.254 \pm 0.054$ & 0.000 \\
\hline OTU222 & & f__Bacteroidaceae & $0.060 \pm 0.033$ & $0.051 \pm 0.017$ & $0.027 \pm 0.013$ & $0.044 \pm 0.014$ & $0.036 \pm 0.015$ & $0.042 \pm 0.015$ & $0.067 \pm 0.023$ & $0.067 \pm 0.018$ & 0.008 \\
\hline OTU110 & & $\begin{array}{l}\text { f__Bacteroidaceae;g__ } \\
\text { Bacteroides }\end{array}$ & $0.383 \pm 0.242$ & $0.292 \pm 0.084$ & $0.185 \pm 0.081$ & $0.395 \pm 0.158$ & $0.186 \pm 0.097$ & $0.355 \pm 0.186$ & $0.436 \pm 0.162$ & $0.641 \pm 0.385$ & 0.007 \\
\hline OTU11 & & $\begin{array}{l}\text { f__Prevotellaceae;g } \\
\text { Prevotella }\end{array}$ & $1.539 \pm 0.626$ & $1.670 \pm 1.444$ & $1.311 \pm 0.684$ & $1.759 \pm 1.014$ & $1.439 \pm 0.896$ & $2.236 \pm 0.544$ & $3.819 \pm 1.587$ & $3.346 \pm 1.413$ & 0.001 \\
\hline OTU66 & & $\begin{array}{l}\text { f__Prevotellaceae;g } \\
\text { Prevotella }\end{array}$ & $0.556 \pm 0.253$ & $0.699 \pm 0.542$ & $0.333 \pm 0.185$ & $0.297 \pm 0.141$ & $0.192 \pm 0.096$ & $0.511 \pm 0.413$ & $1.289 \pm 0.971$ & $0.691 \pm 0.408$ & 0.007 \\
\hline OTU102 & & $\begin{array}{l}\text { f__Prevotellaceae;g } \\
\text { Prevotella }\end{array}$ & $0.247 \pm 0.126$ & $0.178 \pm 0.083$ & $0.198 \pm 0.068$ & $0.139 \pm 0.048$ & $0.184 \pm 0.070$ & $0.124 \pm 0.060$ & $0.368 \pm 0.183$ & $0.469 \pm 0.303$ & 0.001 \\
\hline OTU140 & & $\begin{array}{l}\mathrm{f} \_ \text {Prevotellaceae;g } \\
\text { Prevotella }\end{array}$ & $0.062 \pm 0.017$ & $0.176 \pm 0.137$ & $0.060 \pm 0.046$ & $0.052 \pm 0.025$ & $0.056 \pm 0.020$ & $0.093 \pm 0.059$ & $0.225 \pm 0.173$ & $0.321 \pm 0.191$ & 0.000 \\
\hline OTU146 & & $\begin{array}{l}\text { f__Prevotellaceae;g__ } \\
\text { Prevotella }\end{array}$ & $0.331 \pm 0.266$ & $0.201 \pm 0.082$ & $0.091 \pm 0.042$ & $0.086 \pm 0.039$ & $0.110 \pm 0.051$ & $0.109 \pm 0.023$ & $0.200 \pm 0.044$ & $0.244 \pm 0.061$ & 0.002 \\
\hline OTU154 & & $\begin{array}{l}\text { f__Prevotellaceae;g__ } \\
\text { Prevotella }\end{array}$ & $0.047 \pm 0.034$ & $0.063 \pm 0.056$ & $0.054 \pm 0.037$ & $0.020 \pm 0.032$ & $0.041 \pm 0.047$ & $0.034 \pm 0.034$ & $0.411 \pm 0.320$ & $0.334 \pm 0.403$ & 0.002 \\
\hline OTU224 & & $\begin{array}{l}\text { f__Prevotellaceae;g__ } \\
\text { Prevotella }\end{array}$ & $0.064 \pm 0.036$ & $0.033 \pm 0.020$ & $0.017 \pm 0.014$ & $0.022 \pm 0.016$ & $0.026 \pm 0.014$ & $0.040 \pm 0.037$ & $0.071 \pm 0.030$ & $0.074 \pm 0.059$ & 0.009 \\
\hline
\end{tabular}




\begin{tabular}{|c|c|c|c|c|c|c|c|c|c|c|c|}
\hline OTU & $\mathrm{P}$ & Microbial group & $\mathrm{CT}$ & CTC & $\mathrm{BC}$ & $\mathrm{CBC}$ & $\mathrm{CS}$ & $\mathrm{CO}$ & $\mathrm{COE}$ & $\mathrm{COV}$ & $\begin{array}{c}\mathrm{P} \\
\text { Value }\end{array}$ \\
\hline OTU244 & Bacteroidetes & $\begin{array}{l}\mathrm{f} \_ \text {Prevotellaceae;g } \\
\text { Prevotella }\end{array}$ & $0.049 \pm 0.082$ & $0.015 \pm 0.016$ & $0.041 \pm 0.030$ & $0.020 \pm 0.004$ & $0.027 \pm 0.018$ & $0.007 \pm 0.008$ & $0.046 \pm 0.031$ & $0.107 \pm 0.052$ & 0.002 \\
\hline OTU254 & & $\begin{array}{l}\text { f__Prevotellaceae;g_ } \\
\text { Prevotella }\end{array}$ & $0.037 \pm 0.015$ & $0.031 \pm 0.012$ & $0.018 \pm 0.011$ & $0.022 \pm 0.014$ & $0.035 \pm 0.014$ & $0.031 \pm 0.015$ & $0.165 \pm 0.133$ & $0.068 \pm 0.025$ & 0.000 \\
\hline OTU382 & & $\begin{array}{l}\text { f__Prevotellaceae;g__ } \\
\text { Prevotella }\end{array}$ & $0.030 \pm 0.012$ & $0.014 \pm 0.007$ & $0.014 \pm 0.012$ & $0.009 \pm 0.015$ & $0.017 \pm 0.016$ & $0.004 \pm 0.006$ & $0.019 \pm 0.010$ & $0.033 \pm 0.020$ & 0.006 \\
\hline OTU384 & & $\begin{array}{l}\text { f__Prevotellaceae;g__ } \\
\text { Prevotella }\end{array}$ & $0.026 \pm 0.019$ & $0.014 \pm 0.018$ & $0.005 \pm 0.007$ & $0.002 \pm 0.005$ & $0.002 \pm 0.005$ & $0.010 \pm 0.011$ & $0.034 \pm 0.027$ & $0.019 \pm 0.018$ & 0.006 \\
\hline OTU395 & & $\begin{array}{l}\text { f__Prevotellaceae;g } \\
\text { Prevotella }\end{array}$ & $0.007 \pm 0.010$ & $0.005 \pm 0.009$ & $0.009 \pm 0.010$ & $0.004 \pm 0.009$ & $0.015 \pm 0.017$ & $0.007 \pm 0.013$ & $0.010 \pm 0.011$ & $0.035 \pm 0.012$ & 0.001 \\
\hline OTU442 & & $\begin{array}{l}\text { f__Prevotellaceae;g } \\
\text { Prevotella }\end{array}$ & $0.000 \pm 0.000$ & $0.000 \pm 0.000$ & $0.000 \pm 0.000$ & $0.000 \pm 0.000$ & $0.000 \pm 0.000$ & $0.022 \pm 0.023$ & $0.010 \pm 0.012$ & $0.000 \pm 0.000$ & 0.001 \\
\hline OTU1164 & & $\begin{array}{l}\text { f__Prevotellaceae;g__ } \\
\text { Prevotella }\end{array}$ & $0.000 \pm 0.000$ & $0.000 \pm 0.000$ & $0.000 \pm 0.000$ & $0.000 \pm 0.000$ & $0.000 \pm 0.000$ & $0.000 \pm 0.000$ & $0.007 \pm 0.007$ & $0.000 \pm 0.000$ & 0.000 \\
\hline OTU306 & & $\begin{array}{l}\text { f__Prevotellaceae;g__ } \\
\text { Prevotella;s_copri }\end{array}$ & $0.276 \pm 0.243$ & $0.180 \pm 0.080$ & $0.277 \pm 0.085$ & $0.152 \pm 0.052$ & $0.253 \pm 0.147$ & $0.137 \pm 0.058$ & $0.751 \pm 0.718$ & $0.757 \pm 0.304$ & 0.001 \\
\hline OTU30 & & f__S $24-7$ & $1.241 \pm 1.033$ & $1.541 \pm 1.130$ & $2.919 \pm 1.128$ & $0.854 \pm 0.258$ & $1.961 \pm 1.589$ & $1.456 \pm 0.962$ & $0.545 \pm 0.180$ & $0.765 \pm 0.288$ & 0.003 \\
\hline OTU201 & & f__S $24-7$ & $0.055 \pm 0.080$ & $0.052 \pm 0.043$ & $0.183 \pm 0.135$ & $0.013 \pm 0.011$ & $0.104 \pm 0.074$ & $0.021 \pm 0.013$ & $0.037 \pm 0.020$ & $0.034 \pm 0.035$ & 0.001 \\
\hline OTU424 & & f_ $\quad \mathrm{S} 24-7$ & $0.000 \pm 0.000$ & $0.019 \pm 0.021$ & $0.002 \pm 0.005$ & $0.002 \pm 0.006$ & $0.000 \pm 0.000$ & $0.007 \pm 0.011$ & $0.000 \pm 0.000$ & $0.000 \pm 0.000$ & 0.006 \\
\hline OTU644 & & f__S $24-7$ & $0.018 \pm 0.011$ & $0.024 \pm 0.018$ & $0.029 \pm 0.033$ & $0.023 \pm 0.014$ & $0.029 \pm 0.052$ & $0.162 \pm 0.180$ & $0.030 \pm 0.018$ & $0.010 \pm 0.012$ & 0.010 \\
\hline OTU256 & Firmicutes & $\begin{array}{l}\text { c_Clostridia;o_Clos } \\
\text { tridiales }\end{array}$ & $0.015 \pm 0.003$ & $0.023 \pm 0.008$ & $0.030 \pm 0.011$ & $0.008 \pm 0.014$ & $0.022 \pm 0.015$ & $0.015 \pm 0.010$ & $0.020 \pm 0.004$ & $0.005 \pm 0.009$ & 0.003 \\
\hline OTU263 & & $\begin{array}{l}\text { c_Clostridia;o_Clos } \\
\text { tridiales }\end{array}$ & $0.025 \pm 0.008$ & $0.036 \pm 0.019$ & $0.029 \pm 0.011$ & $0.015 \pm 0.013$ & $0.038 \pm 0.011$ & $0.016 \pm 0.006$ & $0.020 \pm 0.008$ & $0.023 \pm 0.007$ & 0.004 \\
\hline
\end{tabular}




\begin{tabular}{|c|c|c|c|c|c|c|c|c|c|c|c|}
\hline OTU & $\mathrm{P}$ & Microbial group & $\mathrm{CT}$ & CTC & $\mathrm{BC}$ & $\mathrm{CBC}$ & $\mathrm{CS}$ & $\mathrm{CO}$ & $\mathrm{COE}$ & $\mathrm{COV}$ & $\begin{array}{c}\mathrm{P} \\
\text { Value }\end{array}$ \\
\hline OTU273 & Firmicutes & $\begin{array}{l}\text { c_Clostridia;o_Clos } \\
\text { tridiales }\end{array}$ & $0.005 \pm 0.008$ & $0.017 \pm 0.005$ & $0.015 \pm 0.008$ & $0.000 \pm 0.000$ & $0.018 \pm 0.011$ & $0.005 \pm 0.008$ & $0.014 \pm 0.012$ & $0.012 \pm 0.010$ & 0.004 \\
\hline OTU117 & & $\begin{array}{l}\text { c_Clostridia;o_Clos } \\
\text { tridiales }\end{array}$ & $0.099 \pm 0.028$ & $0.133 \pm 0.042$ & $0.159 \pm 0.083$ & $0.073 \pm 0.015$ & $0.095 \pm 0.025$ & $0.169 \pm 0.098$ & $0.085 \pm 0.034$ & $0.054 \pm 0.009$ & 0.003 \\
\hline OTU212 & & $\begin{array}{l}\text { c_Clostridia;o_Clos } \\
\text { tridiales }\end{array}$ & $0.032 \pm 0.034$ & $0.048 \pm 0.046$ & $0.101 \pm 0.035$ & $0.022 \pm 0.018$ & $0.071 \pm 0.042$ & $0.029 \pm 0.030$ & $0.032 \pm 0.017$ & $0.023 \pm 0.027$ & 0.001 \\
\hline OTU70 & & $\mathrm{f} \_[\text {Mogibacteriaceae }]$ & $0.121 \pm 0.054$ & $0.296 \pm 0.191$ & $0.713 \pm 0.707$ & $0.063 \pm 0.021$ & $0.207 \pm 0.052$ & $0.137 \pm 0.103$ & $0.169 \pm 0.091$ & $0.109 \pm 0.070$ & 0.004 \\
\hline OTU1003 & & $\mathrm{f} \_$[Mogibacteriaceae] & $0.002 \pm 0.005$ & $0.011 \pm 0.012$ & $0.015 \pm 0.014$ & $0.000 \pm 0.000$ & $0.012 \pm 0.007$ & $0.011 \pm 0.006$ & $0.009 \pm 0.007$ & $0.022 \pm 0.005$ & 0.003 \\
\hline OTU1125 & & $\mathrm{f} \_$[Mogibacteriaceae] & $0.033 \pm 0.013$ & $0.030 \pm 0.015$ & $0.016 \pm 0.010$ & $0.020 \pm 0.008$ & $0.003 \pm 0.008$ & $0.012 \pm 0.010$ & $0.009 \pm 0.007$ & $0.000 \pm 0.000$ & 0.000 \\
\hline OTU28 & & f_Clostridiaceae & $0.455 \pm 0.301$ & $0.446 \pm 0.126$ & $0.382 \pm 0.109$ & $0.285 \pm 0.076$ & $0.332 \pm 0.114$ & $0.378 \pm 0.129$ & $0.697 \pm 0.386$ & $0.663 \pm 0.165$ & 0.008 \\
\hline OTU58 & & f__Lachnospiraceae & $0.081 \pm 0.080$ & $0.135 \pm 0.086$ & $0.085 \pm 0.033$ & $0.326 \pm 0.217$ & $0.107 \pm 0.070$ & $0.477 \pm 0.334$ & $0.164 \pm 0.110$ & $0.101 \pm 0.059$ & 0.000 \\
\hline OTU312 & & f__Lachnospiraceae & $0.022 \pm 0.019$ & $0.013 \pm 0.007$ & $0.014 \pm 0.008$ & $0.000 \pm 0.000$ & $0.008 \pm 0.007$ & $0.005 \pm 0.007$ & $0.015 \pm 0.008$ & $0.027 \pm 0.010$ & 0.000 \\
\hline OTU367 & & f__Lachnospiraceae & $0.013 \pm 0.021$ & $0.030 \pm 0.027$ & $0.006 \pm 0.009$ & $0.002 \pm 0.006$ & $0.000 \pm 0.000$ & $0.004 \pm 0.006$ & $0.002 \pm 0.005$ & $0.000 \pm 0.000$ & 0.003 \\
\hline OTU315 & & $\begin{array}{l}\text { f_Lachnospiraceae;g } \\
\text { _Coprococcus }\end{array}$ & $0.008 \pm 0.010$ & $0.012 \pm 0.011$ & $0.007 \pm 0.007$ & $0.011 \pm 0.009$ & $0.007 \pm 0.008$ & $0.008 \pm 0.009$ & $0.021 \pm 0.013$ & $0.037 \pm 0.029$ & 0.004 \\
\hline OTU194 & & $\begin{array}{l}\text { f__Lachnospiraceae;g } \\
\text { _Coprococcus;s_cat } \\
\text { us }\end{array}$ & $0.032 \pm 0.004$ & $0.067 \pm 0.041$ & $0.023 \pm 0.005$ & $0.019 \pm 0.017$ & $0.020 \pm 0.009$ & $0.012 \pm 0.010$ & $0.027 \pm 0.014$ & $0.036 \pm 0.013$ & 0.000 \\
\hline OTU48 & & f__Ruminococcaceae & $0.125 \pm 0.043$ & $0.259 \pm 0.160$ & $0.341 \pm 0.109$ & $0.443 \pm 0.242$ & $0.254 \pm 0.069$ & $0.294 \pm 0.107$ & $0.129 \pm 0.031$ & $0.137 \pm 0.075$ & 0.000 \\
\hline OTU120 & & f_Ruminococcaceae & $0.140 \pm 0.080$ & $0.121 \pm 0.043$ & $0.067 \pm 0.010$ & $0.076 \pm 0.042$ & $0.082 \pm 0.025$ & $0.064 \pm 0.018$ & $0.061 \pm 0.013$ & $0.061 \pm 0.019$ & 0.004 \\
\hline OTU200 & & f_Ruminococcaceae & $0.027 \pm 0.015$ & $0.041 \pm 0.012$ & $0.053 \pm 0.014$ & $0.037 \pm 0.023$ & $0.027 \pm 0.007$ & $0.027 \pm 0.010$ & $0.015 \pm 0.008$ & $0.017 \pm 0.011$ & 0.000 \\
\hline OTU326 & & f_Ruminococcaceae & $0.015 \pm 0.014$ & $0.014 \pm 0.015$ & $0.003 \pm 0.009$ & $0.003 \pm 0.007$ & $0.002 \pm 0.005$ & $0.000 \pm 0.000$ & $0.000 \pm 0.000$ & $0.000 \pm 0.000$ & 0.007 \\
\hline OTU808 & & f_Ruminococcaceae & $0.011 \pm 0.009$ & $0.025 \pm 0.010$ & $0.010 \pm 0.008$ & $0.013 \pm 0.011$ & $0.011 \pm 0.011$ & $0.016 \pm 0.011$ & $0.002 \pm 0.005$ & $0.013 \pm 0.002$ & 0.009 \\
\hline OTU13 & & f_Ruminococcaceae & $0.395 \pm 0.153$ & $0.516 \pm 0.380$ & $1.223 \pm 0.835$ & $0.955 \pm 0.475$ & $1.507 \pm 0.840$ & $0.939 \pm 0.643$ & $0.516 \pm 0.292$ & $0.440 \pm 0.161$ & 0.005 \\
\hline
\end{tabular}




\begin{tabular}{|c|c|c|c|c|c|c|c|c|c|c|c|}
\hline OTU & $\mathrm{P}$ & Microbial group & $\mathrm{CT}$ & CTC & $\mathrm{BC}$ & $\mathrm{CBC}$ & $\mathrm{CS}$ & $\mathrm{CO}$ & $\mathrm{COE}$ & $\mathrm{COV}$ & $\begin{array}{c}\mathrm{P} \\
\text { Value }\end{array}$ \\
\hline OTU79 & Firmicutes & f__Ruminococcaceae & $0.047 \pm 0.037$ & $0.126 \pm 0.057$ & $0.203 \pm 0.057$ & $0.058 \pm 0.014$ & $0.096 \pm 0.057$ & $0.155 \pm 0.104$ & $0.090 \pm 0.034$ & $0.051 \pm 0.019$ & 0.000 \\
\hline OTU1066 & & $\begin{array}{l}\text { f__Ruminococcaceae } \\
\text { f__Ruminococcaceae; }\end{array}$ & $0.225 \pm 0.092$ & $0.164 \pm 0.072$ & $0.072 \pm 0.037$ & $0.077 \pm 0.030$ & $0.073 \pm 0.029$ & $0.143 \pm 0.085$ & $0.098 \pm 0.051$ & $0.063 \pm 0.020$ & 0.000 \\
\hline OTU6 & & $\begin{array}{l}\mathrm{g} \_ \text {Faecalibacterium;s } \\
\text { prausnitzii }\end{array}$ & $4.152 \pm 2.776$ & $2.905 \pm 1.050$ & $4.734 \pm 1.975$ & $3.087 \pm 0.880$ & $4.874 \pm 1.550$ & $2.753 \pm 1.059$ & $1.353 \pm 0.386$ & $2.641 \pm 1.411$ & 0.004 \\
\hline OTU16 & & $\begin{array}{l}\text { f__Ruminococcaceae; } \\
\text { g__Oscillospira }\end{array}$ & $0.689 \pm 0.161$ & $1.654 \pm 0.950$ & $1.006 \pm 0.608$ & $0.322 \pm 0.084$ & $0.697 \pm 0.168$ & $0.458 \pm 0.109$ & $0.522 \pm 0.214$ & $0.597 \pm 0.309$ & 0.000 \\
\hline OTU45 & & $\begin{array}{l}\text { f__Ruminococcaceae; } \\
\text { g__Oscillospira }\end{array}$ & $1.021 \pm 0.830$ & $0.638 \pm 0.295$ & $0.627 \pm 0.289$ & $0.262 \pm 0.080$ & $0.428 \pm 0.122$ & $0.274 \pm 0.131$ & $0.251 \pm 0.073$ & $0.360 \pm 0.230$ & 0.004 \\
\hline OTU128 & & $\begin{array}{l}\text { f__Ruminococcaceae; } \\
\text { g__oscillospira }\end{array}$ & $0.077 \pm 0.016$ & $0.201 \pm 0.192$ & $0.053 \pm 0.046$ & $0.044 \pm 0.016$ & $0.029 \pm 0.018$ & $0.059 \pm 0.041$ & $0.040 \pm 0.039$ & $0.022 \pm 0.013$ & 0.004 \\
\hline OTU218 & & $\begin{array}{l}\text { f__Ruminococcaceae; } \\
\text { g__Oscillospira }\end{array}$ & $0.015 \pm 0.014$ & $0.047 \pm 0.041$ & $0.025 \pm 0.007$ & $0.016 \pm 0.011$ & $0.007 \pm 0.008$ & $0.033 \pm 0.015$ & $0.009 \pm 0.011$ & $0.007 \pm 0.010$ & 0.003 \\
\hline OTU220 & & $\begin{array}{l}\text { f_Ruminococcaceae; } \\
\text { g__Oscillospira }\end{array}$ & $0.052 \pm 0.019$ & $0.034 \pm 0.008$ & $0.037 \pm 0.006$ & $0.043 \pm 0.011$ & $0.037 \pm 0.012$ & $0.031 \pm 0.009$ & $0.019 \pm 0.012$ & $0.024 \pm 0.014$ & 0.001 \\
\hline OTU405 & & $\begin{array}{l}\text { f__Ruminococcaceae; } \\
\text { g__Oscillospira }\end{array}$ & $0.018 \pm 0.009$ & $0.014 \pm 0.008$ & $0.005 \pm 0.009$ & $0.005 \pm 0.008$ & $0.004 \pm 0.007$ & $0.002 \pm 0.005$ & $0.000 \pm 0.000$ & $0.000 \pm 0.000$ & 0.000 \\
\hline OTU908 & & $\begin{array}{l}\text { f__Ruminococcaceae; } \\
\text { g__Oscillospira }\end{array}$ & $0.005 \pm 0.007$ & $0.010 \pm 0.009$ & $0.002 \pm 0.006$ & $0.000 \pm 0.000$ & $0.002 \pm 0.005$ & $0.000 \pm 0.000$ & $0.000 \pm 0.000$ & $0.000 \pm 0.000$ & 0.007 \\
\hline OTU15 & & $\begin{array}{l}\text { f__Ruminococcaceae; } \\
\text { g__Ruminococcus }\end{array}$ & $0.252 \pm 0.132$ & $0.305 \pm 0.157$ & $1.410 \pm 0.816$ & $0.595 \pm 0.510$ & $0.651 \pm 0.202$ & $0.438 \pm 0.165$ & $0.190 \pm 0.098$ & $0.210 \pm 0.110$ & 0.000 \\
\hline OTU93 & & $\begin{array}{l}\text { f_Ruminococcaceae; } \\
\text { g__Ruminococcus }\end{array}$ & $0.095 \pm 0.056$ & $0.186 \pm 0.148$ & $0.177 \pm 0.161$ & $0.177 \pm 0.054$ & $0.056 \pm 0.032$ & $0.576 \pm 0.489$ & $0.130 \pm 0.114$ & $0.045 \pm 0.026$ & 0.001 \\
\hline
\end{tabular}




\begin{tabular}{|c|c|c|c|c|c|c|c|c|c|c|c|}
\hline OTU & $\mathrm{P}$ & Microbial group & $\mathrm{CT}$ & CTC & $\mathrm{BC}$ & $\mathrm{CBC}$ & $\mathrm{CS}$ & $\mathrm{CO}$ & $\mathrm{COE}$ & $\mathrm{COV}$ & $\begin{array}{c}\mathrm{P} \\
\text { Value }\end{array}$ \\
\hline OTU153 & & $\begin{array}{l}\text { f__Ruminococcaceae; } \\
\text { g__Ruminococcus }\end{array}$ & $0.046 \pm 0.028$ & $0.105 \pm 0.063$ & $0.066 \pm 0.034$ & $0.113 \pm 0.042$ & $0.025 \pm 0.020$ & $0.276 \pm 0.176$ & $0.069 \pm 0.034$ & $0.022 \pm 0.016$ & 0.000 \\
\hline OTU54 & & $\begin{array}{l}\text { f__Ruminococcaceae; } \\
\text { g__Ruminococcus;s__ } \\
\text { bromii }\end{array}$ & $0.224 \pm 0.079$ & $0.389 \pm 0.105$ & $0.318 \pm 0.056$ & $0.352 \pm 0.183$ & $0.178 \pm 0.063$ & $0.827 \pm 0.514$ & $0.454 \pm 0.080$ & $0.259 \pm 0.147$ & 0.000 \\
\hline OTU17 & & $\begin{array}{l}\mathrm{f} \_ \text {Veillonellaceae;g_ } \\
{ }_{-} \text {Phascolarctobacteriu } \\
\mathrm{m}\end{array}$ & $1.346 \pm 0.505$ & $1.319 \pm 0.554$ & $0.647 \pm 0.168$ & $0.749 \pm 0.352$ & $1.197 \pm 0.508$ & $0.587 \pm 0.362$ & $0.656 \pm 0.361$ & $0.660 \pm 0.481$ & 0.004 \\
\hline OTU170 & Proteobacteria & $\begin{array}{l}\mathrm{f} \_ \text {Alcaligenaceae;g__ } \\
\text { Sutterella }\end{array}$ & $0.027 \pm 0.024$ & $0.021 \pm 0.012$ & $0.073 \pm 0.049$ & $0.033 \pm 0.014$ & $0.069 \pm 0.038$ & $0.025 \pm 0.010$ & $0.028 \pm 0.022$ & $0.037 \pm 0.016$ & 0.005 \\
\hline OTU317 & & $\begin{array}{l}\mathrm{f} \_ \text {Xanthomonadaceae } \\
; \mathrm{g} \_ \text {Stenotrophomona } \\
\mathrm{s}\end{array}$ & $0.021 \pm 0.013$ & $0.023 \pm 0.009$ & $0.029 \pm 0.006$ & $0.008 \pm 0.013$ & $0.035 \pm 0.027$ & $0.012 \pm 0.009$ & $0.014 \pm 0.013$ & $0.004 \pm 0.006$ & 0.003 \\
\hline
\end{tabular}


Table S1 Raw reads, Effective Tags and OTUs from each groups

\begin{tabular}{ccccc}
\hline Groups & Raw reads & Effective Tags & OTUs & Average Length \\
\hline CT & 85468 & 57237 & 689 & 413 \\
CTC & 80596 & 53948 & 667 & 413 \\
BC & 76418 & 50169 & 636 & 413 \\
CBC & 83335 & 55040 & 670 & 413 \\
CS & 79889 & 52676 & 663 & 414 \\
CO & 80874 & 52920 & 663 & 413 \\
COE & 79605 & 50792 & 608 & 416 \\
COV & 77326 & 49767 & 626 & 416 \\
\hline
\end{tabular}

CT: Control group; CTC: Chlortetracycline; BC: bacitracin zinc and colistin sulphate; $\mathrm{CBC}$ : chlortetracycline, bacitracin zinc and colistin sulphate; CS: chlortetracycline and Macleaya cordata extracts; CO: chlortetracycline and olaquindox; COE: chlortetracycline, olaquindox and enramycin; COV: chlortetracycline, olaquindox and virginiamycin. 
Table S3 Effect of each group on the relative proportion (percentage \pm standard deviation of the mean) of the most abundant phyla and genera in the each treatment pigs ( $\mathrm{n}=6$ ).

\begin{tabular}{|c|c|c|c|c|c|c|c|c|}
\hline Taxon & $\mathrm{CT}$ & CTC & $\mathrm{BC}$ & $\mathrm{CBC}$ & $\mathrm{CS}$ & $\mathrm{CO}$ & $\mathrm{COE}$ & $\mathrm{COV}$ \\
\hline \multicolumn{9}{|l|}{ Phylum } \\
\hline Firmicutes & $51.8 \pm 3.2$ & $59.4 \pm 1.6^{*}$ & $60.4 \pm 1.1^{*}$ & $57.1 \pm 2.5$ & $56.0 \pm 1.9$ & $57.7 \pm 3.9$ & $47.0 \pm 2.5$ & $43.7 \pm 1.0^{*}$ \\
\hline Bacteroidetes & $38.1 \pm 0.6$ & $35.2 \pm 1.3$ & $36.9 \pm 1.1$ & $38.9 \pm 2.2$ & $38.5 \pm 1.7$ & $36.5 \pm 2.3$ & $49.7 \pm 2.3^{* *}$ & $49.6 \pm 0.8 * *$ \\
\hline Proteobacteria & $7.6 \pm 3.1$ & $2.7 \pm 0.7^{*}$ & $1.8 \pm 0.3 * *$ & $2.6 \pm 0.9 *$ & $3.9 \pm 1.4$ & $3.0 \pm 1.4^{*}$ & $1.9 \pm 0.3 * *$ & $5.1 \pm 1.5$ \\
\hline Euryarchaeota & $0.72 \pm 0.41$ & $1.32 \pm 1.20$ & $0.03 \pm 0.01$ & $0.08 \pm 0.06$ & $0.32 \pm 0.29$ & $1.06 \pm 0.94$ & $0.10 \pm 0.09$ & $0.12 \pm 0.09$ \\
\hline Spirochaetes & $0.65 \pm 0.44$ & $0.34 \pm 0.13$ & $0.11 \pm 0.02 *$ & $0.19 \pm 0.09$ & $0.24 \pm 0.06$ & $0.35 \pm 0.19$ & $0.18 \pm 0.04$ & $0.24 \pm 0.08$ \\
\hline Tenericutes & $0.21 \pm 0.03$ & $0.32 \pm 0.12$ & $0.19 \pm 0.04$ & $0.37 \pm 011$ & $0.35 \pm 0.15$ & $0.65 \pm 0.30$ & $0.54 \pm 0.21$ & $0.36 \pm 0.12$ \\
\hline Cyanobacteria & $0.51 \pm 0.29$ & $0.24 \pm 0.07$ & $0.25 \pm 0.13$ & $0.40 \pm 0.14$ & $0.23 \pm 0.07$ & $0.32 \pm 0.08$ & $0.27 \pm 0.08$ & $0.47 \pm 0.16$ \\
\hline Actinobacteria & $0.43 \pm 0.03$ & $0.43 \pm 0.05$ & $0.35 \pm 0.03$ & $0.26 \pm 0.02 * *$ & $0.40 \pm 0.06$ & $0.31 \pm 0.04 *$ & $0.23 \pm 0.02 * *$ & $0.26 \pm 0.03 * *$ \\
\hline Elusimicrobia & $0.054 \pm 0.043$ & $0.016 \pm 0.006$ & $0.005 \pm 0.002$ & $0.006 \pm 0.002$ & $0.012 \pm 0.006$ & $0.029 \pm 0.022$ & $0.011 \pm 0.006$ & $0.011 \pm 0.003$ \\
\hline Fibrobacteres & $0.0000 \pm 0.0000$ & $0.0012 \pm 0.0012$ & $0.0012 \pm 0.0012$ & $0.0015 \pm 0.0008$ & $0.0004 \pm 0.0004$ & $0.0251 \pm 0.0200$ & $0.0031 \pm 0.0023$ & $0.0027 \pm 0.0019$ \\
\hline Others & $0.027 \pm 0.012$ & $0.030 \pm 0.006$ & $0.026 \pm 0.005$ & $0.043 \pm 0.021$ & $0.054 \pm 0.019$ & $0.032 \pm 0.007$ & $0.042 \pm 0.006$ & $0.078 \pm 0.038$ \\
\hline \multicolumn{9}{|l|}{ Genus } \\
\hline Prevotella & $15.8 \pm 1.4$ & $12.3 \pm 1.7$ & $15.1 \pm 1.5$ & $16.0 \pm 2.9$ & $18.1 \pm 2.6$ & $14.5 \pm 1.1$ & $30.7 \pm 2.1 * *$ & $28.6 \pm 3.1 * *$ \\
\hline Roseburia & $6.1 \pm 1.0$ & $11.6 \pm 3.1$ & $10.7 \pm 0.9$ & $8.5 \pm 3.6$ & $9.0 \pm 1.0$ & $6.8 \pm 2.2$ & $8.0 \pm 1.7$ & $6.9 \pm 1.3$ \\
\hline Succinivibrio & $7.1 \pm 3.2$ & $2.3 \pm 0.7^{*}$ & $1.3 \pm 0.3^{* *}$ & $2.2 \pm 0.9^{*}$ & $3.4 \pm 1.4$ & $2.7 \pm 1.3^{*}$ & $1.6 \pm 0.3^{*}$ & $4.8 \pm 1.5$ \\
\hline [Prevotella] & $6.7 \pm 2.1$ & $5.0 \pm 1.5$ & $5.8 \pm 0.6$ & $4.7 \pm 0.7$ & $4.8 \pm 0.9$ & $5.3 \pm 0.6$ & $6.0 \pm 0.7$ & $4.7 \pm 0.4$ \\
\hline Faecalibacterium & $4.7 \pm 1.2$ & $3.6 \pm 0.4$ & $6.0 \pm 1.1$ & $3.8 \pm 0.4$ & $5.6 \pm 0.5$ & $3.9 \pm 0.8$ & $1.9 \pm 0.3 * *$ & $3.3 \pm 0.7$ \\
\hline Lachnospira & $3.3 \pm 0.6$ & $2.6 \pm 0.2$ & $3.7 \pm 0.7$ & $2.5 \pm 0.2$ & $3.4 \pm 0.4$ & $3.3 \pm 0.8$ & $2.8 \pm 0.3$ & $3.3 \pm 0.3$ \\
\hline Methanobrevibacter & $0.72 \pm 0.41$ & $1.30 \pm 1.20$ & $0.03 \pm 0.01$ & $0.07 \pm 0.06$ & $0.32 \pm 0.29$ & $1.06 \pm 0.94$ & $0.10 \pm 0.09$ & $0.12 \pm 0.09$ \\
\hline Ruminococcus & $2.1 \pm 0.2$ & $2.3 \pm 0.2$ & $3.4 \pm 0.4^{*}$ & $4.3 \pm 0.8^{* *}$ & $2.9 \pm 0.1$ & $4.0 \pm 0.5^{* *}$ & $2.5 \pm 0.2$ & $2.3 \pm 0.2$ \\
\hline CF231 & $1.74 \pm 0.90$ & $0.94 \pm 0.08$ & $0.89 \pm .0 .09$ & $0.62 \pm 0.11$ & $0.75 \pm 0.19$ & $0.79 \pm 0.13$ & $0.86 \pm 0.09$ & $0.88 \pm 0.12$ \\
\hline Oscillospira & $3.4 \pm 0.5$ & $4.3 \pm 0.5$ & $3.6 \pm 0.5$ & $2.0 \pm 0.2 *$ & $2.6 \pm 0.1^{*}$ & $2.3 \pm 0.3$ & $2.0 \pm 0.3$ & $2.2 \pm 0.3$ \\
\hline Others & $48.2 \pm 4.0$ & $53.8 \pm 3.8$ & $49.4 \pm 2.2$ & $55.4 \pm 6.2$ & $49.0 \pm 3.3$ & $55.3 \pm 4.3$ & $43.5 \pm 3.2$ & $42.9 \pm 3.5$ \\
\hline
\end{tabular}

CT: Control group; CTC: Chlortetracycline; BC: bacitracin zinc and colistin sulphate; CBC: chlortetracycline, bacitracin zinc and colistin sulphate; CS: chlortetracycline and Macleaya cordata extracts; CO: chlortetracycline and olaquindox; COE: chlortetracycline, olaquindox and enramycin; COV: chlortetracycline, olaquindox and virginiamycin. The relative abundances of phyla and genera are presented as mean \pm SEM. Statistical significance was analyzed by the Student's t tests. Statistically significant comparison results are marked with $* P<0.05$, and $* * P<0.01$. 
Table S4. OTU Taxonomy.

\begin{tabular}{|c|c|}
\hline OTU_num & Taxonomy \\
\hline OTU1 & Clostridia;o__Clostridiales;f_Ruminococcaceae \\
\hline OTU1003 & 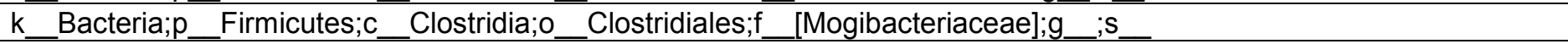 \\
\hline OTU1066 & 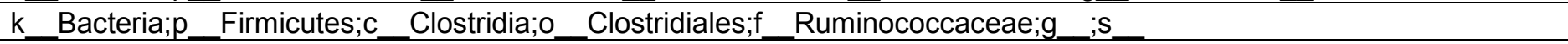 \\
\hline OTU1099 & k__Bacteria;p_Firmicutes;c__Clostridia;o__Clostridiales;f__Lachnospiraceae;g_Roseburia;s_faecis \\
\hline OTU11 & k_Bacteria;p_Bacteroidetes;c__Bacteroidia;o_Bacteroidales;f_Prevotellaceae;g_Prevotella;s_ \\
\hline OTU110 & 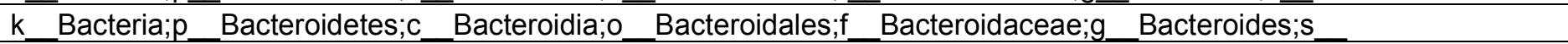 \\
\hline OTU117 & 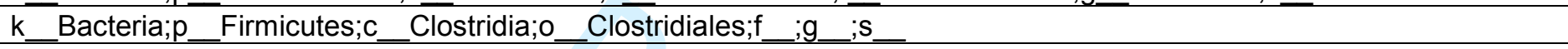 \\
\hline OTU120 & 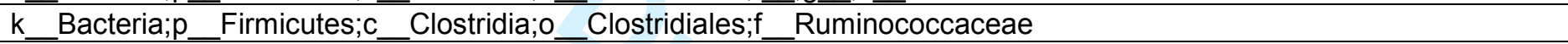 \\
\hline OTU128 & 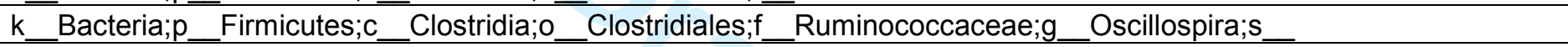 \\
\hline OTU13 & 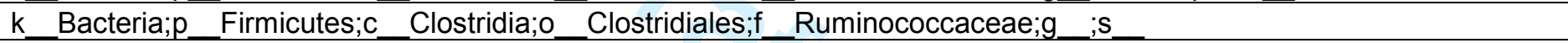 \\
\hline OTU14 & k_Bacteria;p_Bacteroidetes;C__Bacteroidia;o_Bacteroidales;f_[Paraprevotellaceae];g_[Prevotella];s_ \\
\hline OTU140 & k_Bacteria; $p \_$Bacteroidetes; __Bacteroidia; o_Bacteroidales;f_Prevotellaceae;g_Prevotella;s \\
\hline OTU16 & 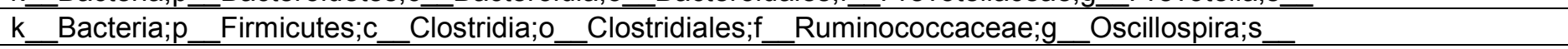 \\
\hline OTU17 & k_Bacteria;p_Firmicutes;c_Clostridia;o_Clostridiales;f_Veillonellaceae;g_Phascolarctobacterium;s \\
\hline OTU170 & k_Bacteria; $p \_$Proteobacteria; $c$ _Betaproteobacteria;o_Burkholderiales;f_Alcaligenaceae; $g$ _Sutterella;s \\
\hline OTU18 & Bacteria;p_Bacteroidetes;c__Bacteroidia;o__Bacteroidales;f_Prevotellaceae;g__Prevotella;s__ \\
\hline OTU19 & 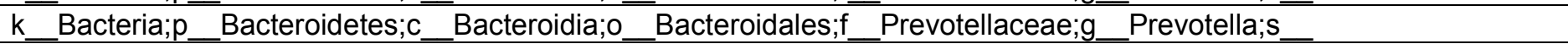 \\
\hline OTU194 & Bacteria;p_Firmicutes;c_Clostridia;o_Clostridiales;f_Lachnospiraceae;g_Coprococcus;s_catus \\
\hline OTU2 & 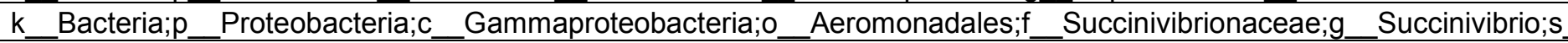 \\
\hline OTU200 & Bacteria;p__Firmicutes;c_Clostridia;o__Clostridiales;f_Ruminococcaceae \\
\hline OTU201 & k_Bacteria;p_Bacteroidetes;c_Bacteroidia;o_Bacteroidales;f_s24-7;g_;s \\
\hline OTU21 & k_Bacteria;p_Bacteroidetes;c_B_Bacteroidia;o_Bacteroidales;f_s24-7;g_;s \\
\hline
\end{tabular}




\begin{tabular}{|c|c|c|c|}
\hline OTU212 & $\mathrm{k}$ & Bacteria;p & Firmicutes;c_Clostridia;o__Clostridiales; $f_{\ldots} ; g_{2} ; \mathrm{s}$ \\
\hline OTU214 & $\mathrm{k}$ & Bacteria; & Bacteroidetes;c__ Bacteroidia;o__Bacteroidales;f__Prevotellaceae;g__Prevotella;s \\
\hline OTU218 & $\mathrm{k}$ & Bacteria;p & Firmicutes;c Clostridia;o Clostridiales;f Ruminococcaceae;g Oscillospira;s \\
\hline OTU220 & $\mathrm{k}$ & Bacteria;p & Firmicutes;c_Clostridia;o_ Clostridiales;f_ Ruminococcaceae;g_ Oscillospira;s \\
\hline OTU222 & $\mathrm{k}$ & Bacteria; & Bacteroidetes;c__Bacteroidia;o__ Bacteroidales;f__ Bacteroidaceae \\
\hline OTU224 & $\mathrm{k}$ & Bacteria;p & Bacteroidetes;c__ Bacteroidia;o__Bacteroidales;f__Prevotellaceae;g__Prevotella;s \\
\hline OTU244 & $\mathrm{k}$ & Bacteria;p & Bacteroidia;o__Bacteroidales;f__Prevotellaceae;g__Prevotella;s \\
\hline OTU254 & $\mathrm{k}$ & Bacteria;p & Bacteroidetes;c__ Bacteroidia;o__Bacteroidales;f__Prevotellaceae;g_ Prevotella;s \\
\hline OTU256 & $\mathrm{k}$ & Bacteria;p & Firmicutes;c Clostridia;o Clostridiales \\
\hline OTU263 & $\mathrm{k}$ & Bacteria; & Firmicutes;c_Clostridia;o__ Clostridiales \\
\hline OTU27 & $\mathrm{k}$ & Bacteria;p & Bacteroidetes;c__Bacteroidia;o__Bacteroidales;f__Prevotellaceae;g__Prevotella;s__copri \\
\hline OTU273 & $\mathrm{k}$ & Bacteria;p & Firmicutes;c_Clostridia;o _ Clostridiales \\
\hline OTU276 & $\mathrm{k}$ & Bacteria;p & Actinobacteria;c__Coriobacteriia;o__Coriobacteriales;f__Coriobacteriaceae;g_;s_ \\
\hline OTU28 & $\mathrm{k}$ & Bacteria;p & Firmicutes;c Clostridia;o Clostridiales;f Clostridiaceae;g ;s \\
\hline OTU281 & $\mathrm{k}$ & Bacteria;p & Actinobacteria;c_C_Coriobacteriia;o__Coriobacteriales;f_Coriobacteriaceae;g__Slackia;s \\
\hline OTU3 & $\mathrm{k}$ & Bacteria; & Firmicutes;c_Clostridia;o__ Clostridiales;f__Lachnospiraceae;g_ Roseburia;s \\
\hline OTU30 & $\mathrm{k}$ & Bacteria;p & Bacteroidetes;c Bacteroidia;o Bacteroidales;f S24-7;g_; \\
\hline OTU30 & $\mathrm{k}$ & Bacteria;p & Bacteroidetes; $\mathrm{C}$ _B Bacteroidia;o_ \\
\hline OTU306 & $\mathrm{k}$ & Bacteria; $p_{-}$ & Bacteroidetes;c__Bacteroidia;o__Bacteroidales;f__Prevotellaceae;g__Prevotella;s \\
\hline OTU312 & $\mathrm{k}$ & Bacteria;p & Firmicutes;c_Clostridia;o__ Clostridiales;f__Lachnospiraceae \\
\hline OTU315 & $\mathrm{k}$ & Bacteria;p & Firmicutes;C__Clostridia;o__Clostridiales;f__Lachnospiraceae;g__Coprococcus;s__ \\
\hline OTU317 & $\mathrm{k}$ & Bacteria;p & Proteobacteria;c_ Gammaproteobacteria;o__ Xanthomonadales;f__ Xanthomonadaceae;g_ Stenotrophomonas;s \\
\hline OTU32 & $\mathrm{k}$ & Bacteria; $p_{2}$ & Bacteroidetes;c__Bacteroidia;o__Bacteroidales;f__[Paraprevotellaceae];g_ [Prevotella];s_ \\
\hline OTU326 & $\mathrm{k}$ & Bacteria;p & Firmicutes;c__Clostridia;o__Clostridiales;f__Ruminococcaceae \\
\hline OTU34 & $\mathrm{k}$ & Bacteria; $p$ & Bacteroidetes;c_ Bacteroidia;o_ Bacteroidales;f_ Prevotellaceae;g \\
\hline OTU367 & $\mathrm{k}$ & Bacteria;p & Firmicutes;C__Clostridia;o__Clostridiales;f__Lachnospiraceae \\
\hline OTU382 & $\mathrm{k}$ & Bacteria;p & Bacteroidetes;c Bacteroidia;o Bacteroidales;f Prevotellaceae;g Prevotella;s \\
\hline OTU384 & $\mathrm{k}$ & Bacteria; $p_{2}$ & Bacteroidetes;c__Bacteroidia;o__Bacteroidales;f__Prevotellaceae;g_ Prevotella;s \\
\hline OTU395 & $\mathrm{k}$ & Bacteria; $p_{-}$ & Bacteroidetes;c__Bacteroidia;o__Bacteroidales;f__Prevotellaceae;g_ \\
\hline OTU4 & $\mathrm{k}$ & Bacteria;p & Firmicutes;c_Clostridia;o_ Clostridiales;f__Lachnospiraceae \\
\hline OTU405 & $\mathrm{k}$ & Bacteria;p & Firmicutes;c__Clostridia;o___Clostridiales;f__Ruminococcaceae;g__Oscillospira;s \\
\hline OTU424 & $\mathrm{k}$ & Bacteria;p & Bacteroidetes;c__Bacteroidia;o__Bacteroidales;f__s24-7;g_;s_ \\
\hline OTU442 & $\mathrm{k}$ & Bacteria; & Bacteroidetes;c__Bacteroidia;o__Bacteroidales;f__Prevotellaceae;g__Prevotella;s \\
\hline
\end{tabular}




\begin{tabular}{|c|c|c|c|}
\hline OTU45 & $\mathrm{k}$ & Bacteria; $p_{-}$ & _Clostridia;o__Clostridiales;f \\
\hline OTU48 & $\mathrm{k}$ & Bacteria;p & Firmicutes;c_Clostridia;o__Clostridiales;f \\
\hline OTU5 & $\mathrm{k}$ & Bacteria; & Firmicutes;c_Clostridia;o__ Clostridiales;f_Lachnospiraceae;g \\
\hline OTU51 & $\mathrm{k}$ & Bacteria;p & Bacteroidetes;c__Bacteroidia;o__Bacteroidales;f__[Paraprevotellaceae];g__[Prevotella];s_ \\
\hline OTU54 & $\mathrm{k}$ & Bacteria;p & Firmicutes;c__Clostridia;o__Clostridiales;f__Ruminococcaceae;g__Ruminococcus;s_bromii \\
\hline OTU58 & $\mathrm{k}$ & Bacteria;p & Firmicutes;c__Clostridia;o__Clostridiales;f__Lachnospiraceae \\
\hline OTU6 & $\mathrm{k}$ & Bacteria;p & Clostridiales;f__Ruminococcaceae;g__Faecalibacterium;s_ \\
\hline OTU62 & $\mathrm{k}$ & Bacteria; & Firmicutes;c_Clostridia;o_ Clostridiales;f__ Ruminococcaceae;g_; \\
\hline OTU644 & $\mathrm{k}$ & Bacteria;p & Bacteroidetes;c__Bacteroidia;o__Bacteroidales;f__s24-7;g_;s_ \\
\hline OTU66 & $\mathrm{k}$ & Bacteria;p & Bacteroidetes;c__Bacteroidia;o__Bacteroidales;f__Prevotellaceae;g__Prevotella;s \\
\hline OTU7 & $\mathrm{k}$ & Bacteria;p & Bacteroidetes;c__Bacteroidia;o__Bacteroidales \\
\hline OTU70 & $\mathrm{k}$ & Bacteria; $p$ & Firmicutes;c__Clostridia;o__Clostridiales;f__[Mogibacteriaceae];g__; _ _ \\
\hline OTU73 & $\mathrm{k}$ & Bacteria; & Bacteroidetes;c__Bacteroidia;o__Bacteroidales;f__[Paraprevotellaceae];g_ ;s \\
\hline OTU79 & $\mathrm{k}$ & Bacteria;p & Firmicutes;c__Clostridia;o__Clostridiales;f__Ruminococcaceae;g_ ;s_ \\
\hline OTU808 & $\mathrm{k}$ & Bacteria; & Firmicutes;c__Clostridia;o__Clostridiales;f__Ruminococcaceae \\
\hline OTU82 & $\mathrm{k}$ & Bacteria;p & Bacteroidetes;c__Bacteroidia;o__Bacteroidales;f_;g_;s__ \\
\hline OTU9 & $\mathrm{k}$ & Bacteria;p & Bacteroidetes;c__Bacteroidia;o__Bacteroidales;f__s24-7;g__s \\
\hline OTU908 & $\mathrm{k}$ & Bacteria;p & Firmicutes;c__Clostridia;o__Clostridiales;f__Ruminococcaceae;g_ \\
\hline OTU93 & $\mathrm{k}$ & Bacteria;p & Clostridiales;f__ Ruminococcaceae;g_ \\
\hline
\end{tabular}

